\title{
Vortices for a variational problem related to superconductivity
}

\author{
by
}

Fabrice BETHUEL

Université Paris-Sud, Laboratoire d'Analyse Numérique, URA-760, Bâtiment 425, 91405 Orsay Cedex, France.

and

\section{Tristan RIVIÈRE}

École Normale Supérieure de Cachan, Centre de Mathématiques et de leurs Applications, Unité associée au CNRS URA-1611, 61, avenue du président Wilson, 94235 Cachan Cedex, France.

ABSTRACT. - We study minimizers of Ginzburg-Landau functionals, which depend on a parameter $\epsilon$. These functionals appear in superconductivity and two dimensional abelian Higgs models. We study the asymptotic limit, as $\epsilon \rightarrow 0$, of minimizers and show that the limiting configuration has vortices, which have topological degree one.

RÉsuMÉ. - Nous étudions les applications minimisantes de la fonctionnelle de Ginzburg-Landau dépendant d'un paramètre $\epsilon$. Cette fonctionnelle intervient dans les problèmes de supraconductivité ainsi que dans le modèle abélien de Higgs en dimension deux. Plus particulièrement, nous étudions le comportement asymptotique de ces applications minimisantes lorsque $\epsilon$ tend vers 0 et nous montrons que la configuration limite a des tourbillons de degré 1 .

\section{INTRODUCTION}

Let $\Omega$ be a simply connected bounded domain in $\mathbb{R}^{2}$. Let $\epsilon>0$ be a parameter and $u_{0}: \partial \Omega \rightarrow S^{1}$ be a smooth map. In a recent book, 
F. Bethuel, H. Brezis and F. Hélein [2] (see also [1], [3], and [4]) have studied the functional

$$
F_{\epsilon}(u)=\frac{1}{2} \int_{\Omega}|\nabla u|^{2}+\frac{1}{4 \epsilon^{2}} \int_{\Omega}\left(1-|u|^{2}\right)^{2}
$$

for $u \in H_{u_{0}}^{1}\left(\Omega, \mathbb{R}^{2}\right)$. Let $u_{\epsilon}$ be a minimizer of $F_{\epsilon}(u)$ on $H_{u_{0}}^{1}\left(\Omega, \mathbb{R}^{2}\right)$. They have studied the asymptotic limit $\epsilon \rightarrow 0$ of $u_{\epsilon}$ and proved that $u_{\epsilon}$ converges to a limiting map $u_{*}$, which has exactly $|d|$ singularities (vortices), where $d=\operatorname{deg}(u, \partial \Omega)$. Moreover, the distribution of the singularities was characterized, and the map $u_{*}$ identified. They also proved that $u_{\epsilon}$ converges to $u_{*}$ in $C_{l o c}^{1}$, away from the singularities.

Functionals of the type of $F_{\epsilon}$ arises in many problems in low temperature physics, for instance superfluidity. Our purpose in this paper is to extend the methods and results of [2] (see also H. Brezis, F. Merle and T. Riviere [5] and [6]) to related functionals arising in superconductivity and two-dimensional abelian Higgs models. More precisely, consider the functional

$$
G_{\epsilon}(u, A)=\frac{1}{2} \int_{\Omega}|\nabla u-i A u|^{2}+\frac{1}{4 \epsilon^{2}} \int_{\Omega}\left(1-|u|^{2}\right)^{2}+\frac{1}{2} \int_{\Omega}|d A|^{2},
$$

where $u \in H^{1}\left(\Omega, R^{2}\right)$, and $A$ (the vector potential), is a real valued 1 -form, that is

$$
A=A_{1} d x_{1}+A_{2} d x_{2} \text {. }
$$

The main characteristic of $G_{\epsilon}(u, A)$ is its invariance under gauge transformations. More precisely let $\phi$ be a function in $H^{2}(\Omega, \mathbb{R})$ and consider

$$
\left\{\begin{array}{l}
u_{\phi}=e^{i \phi} u \\
A_{\phi}=A+d \phi
\end{array}\right.
$$

We have

$$
G_{\epsilon}\left(u_{\phi}, A_{\phi}\right)=G_{\epsilon}(u, A)
$$

The functional $G$ was introduced by Ginzburg and Landau in 1950, in their study of phase transitions, for superconductors. The gauge invariance of the functional allows to account for electromagnetic effects. In particular

$$
h=\star d A
$$

is the induced magnetic field. Moreover physically relevant quantities, are those which are, as $h$, gauge invariant, for instance $|u|$, the density of 
supraconductor), $J=\left(i u, d_{A} u\right)$ (the current), where $d_{A} u=d u-i A u$, and the degree of $u$ on the boundary if we assume for instance $|u|=1$ on the boundary (see below).

In order to have a well posed minimization problem, we need to supplement the functional with a boundary condition. Clearly a Dirichlet type boundary condition (as for $F_{\epsilon}$ ) is not consistent with the gauge invariance. We adopt instead the following approach.

Let $d>0$, be an integer, and $g: \partial \Omega \rightarrow \mathbb{R}$ be a smooth given function. We assume throughout the paper that $\Omega$ is simply connected. Consider the space

$$
V=\left\{\begin{array}{c}
(u, A) \in H^{1}\left(\Omega, \mathbb{R}^{2}\right) \times H^{1}\left(\Omega, \mathbb{R}^{2}\right), \text { such that } \\
|u|=1 \text { on } \partial \Omega \\
J . \tau \equiv\left(i u, \tau \cdot\left(\nabla_{A} u\right)\right)=g \\
\operatorname{deg}(u, \partial \Omega)=d .
\end{array}\right\}
$$

Here $\tau$ denotes the unit tangent vector to $\partial \Omega$, such that $(\nu, \tau)$ is direct, $\nu$ denoting the exterior normal to $\partial \Omega$, and $\nabla_{A} u=\nabla u-i A u$. We recall that $\partial \Omega$ is connected, since $\Omega$ is assumed simply connected. The boundary condition $J . \tau \equiv\left(i u, \tau . \nabla_{A} u\right)$ can be written as

$$
\partial_{\tau} u=(g+A . \tau) i u \quad \text { on } \partial \Omega .
$$

Since the r.h.s. of (7) is clearly in $L^{2}$ (by the trace theorem, and the assumption $g \in C^{1}(\partial \Omega)$ ), we see that if (7) is satisfied $u \in H^{1}\left(\partial \Omega, S^{1}\right)$, for $u \in V$. In particular

$$
\operatorname{deg}(u, \partial \Omega)=\frac{1}{2 \pi} \int_{\partial \Omega} u \times u_{\tau} d \tau
$$

is well defined (one could also invoke instead a result in [7], which shows that the degree is well defined in $\left.H^{\frac{1}{2}}\left(\partial \Omega, S^{1}\right)\right)$.

THEOREM 1. - We have

$$
\underset{(u, A) \in V}{\operatorname{Inf}} G_{\epsilon}(u, A)
$$

is achieved.

In view of the gauge invariance, once we have a minimizer we have actually a whole family $\left(u_{\phi}, A_{\phi}\right)$ of minimizers. It is convenient to reduce this degeneracy by imposing the condition

$$
\left\{\begin{array}{l}
d^{*} A=0 \quad \text { in } \quad \Omega \\
A \cdot \nu=0 \quad \text { on } \quad \partial \Omega .
\end{array}\right.
$$

Vol. $12, \mathrm{n}^{\circ} 3-1995$. 
We have

THEOREM 2. - There is a minimizer $\left(u_{\epsilon}, A_{\epsilon}\right)$ of (8) satisfying

$$
\left\{\begin{array}{l}
d^{*} A_{\epsilon}=0 \quad \text { in } \quad \Omega \\
A_{\epsilon} \cdot \nu=0 \quad \text { on } \quad \partial \Omega
\end{array}\right.
$$

Carrying out an asymptotic analysis as in [2], we are able to prove the following, which is our main result

THEOREM 3. - Let $\left(u_{\epsilon}, A_{\epsilon}\right)$ be minimizers of (8), satifying (9). There is a subsequence $\epsilon_{n} \rightarrow 0$ and exactly $d$ points $a_{1}, a_{2}, \ldots, a_{d}$ in $\Omega$ and $\left(u_{*}, A_{*}\right)$ smooth except at the points $a_{1}, a_{2}, \ldots, a_{d}$ such that

$$
\begin{gathered}
\begin{cases}d^{*} A_{*}=0 & \text { in } \quad \Omega \\
A_{*} \cdot \nu=0 & \text { on } \quad \partial \Omega,\end{cases} \\
u_{\epsilon_{n}} \rightarrow u_{*} \text { strongly in } H_{l o c}^{1}\left(\Omega \backslash \bigcup_{i=1}^{d}\left\{a_{i}\right\}\right) \text { and } W^{1, p}(\Omega) \text { for } p<2 .
\end{gathered}
$$

Set $h_{\epsilon_{n}}=\star d A_{\epsilon_{n}}$ and $h_{*}=\star d A_{*}$. We have

$$
h_{\epsilon_{n}} \rightarrow h_{*} \text { strongly in } H_{l o c}^{1}\left(\Omega \backslash \bigcup_{i=1}^{d}\left\{a_{i}\right\}\right) \text { and } W^{1, p}(\Omega) \text { for } p<2 \text {, }
$$

and $h_{*}$ satified the equation

$$
\left\{\begin{array}{l}
-\Delta h_{*}+h_{*}=2 \pi \sum_{i=1}^{n} \delta_{a_{i}} \quad \text { in } \Omega \\
\frac{\partial h_{*}}{\partial \nu}=-g \quad \text { on } \partial \Omega .
\end{array}\right.
$$

Once the points $a_{i}$ are determined, we are able to recover all informations from the equation (13) which is referred to in the physical litterature as the London equation. Let $\xi_{*}$ be the solution of

$$
\left\{\begin{array}{l}
\Delta \xi_{*}=h_{*} \\
\xi_{*}=0 \quad \text { on } \quad \partial \Omega
\end{array}\right.
$$

We have

$$
\begin{aligned}
& A_{*}=\star d \xi_{*} \\
& J_{*}=\left(i u_{*}, d_{A_{*}} u_{*}\right)=-\star d h .
\end{aligned}
$$


Finally $u_{*}$ is determined (up to a rotation) by

$$
u_{*}=\prod_{k=1}^{d} \frac{z-a_{k}}{\left|z-a_{k}\right|} e^{i \phi},
$$

where $\phi$ is an harmonic function satisfying

$$
\left\{\begin{array}{lll}
\Delta \phi=0 & \text { in } & \Omega \\
\phi=\phi_{0} & \text { on } & \partial \Omega
\end{array}\right.
$$

where $\phi_{0}$ satisfies on $\partial \Omega$

$$
\frac{\partial \phi_{0}}{\partial \tau}=-\left(i \prod_{k=1}^{d} \frac{z-a_{k}}{\left|z-a_{k}\right|}, \frac{\partial}{\partial \tau}\left(\prod_{k=1}^{d} \frac{z-a_{k}}{\left|z-a_{k}\right|}\right)\right)+\frac{\partial \xi}{\partial \nu}+g
$$

( $\phi_{0}$ is uniquely determined up to a constant, this is also the case for $\phi$ ).

Finally the configuration $\left\{a_{i}\right\}$ is governed by a renormalized energy, which is very similar to the one introduced in [2]. Consider a configuration $b=\left(b_{1}, \ldots, b_{d}\right)$ of $d$ distinct points of $\Omega$ and the function

$$
\begin{aligned}
W(b)= & -\pi \sum_{i \neq j} \log \left|b_{i}-b_{j}\right|-\pi \sum_{i=1}^{d} R\left(b_{i}\right)+\pi \sum_{i=1}^{d} \xi_{*}\left(b_{i}\right) \\
& +\frac{1}{2} \int_{\partial \Omega} \Phi\left(g+\frac{\partial \xi}{\partial \nu}\right)+\frac{1}{2} \int_{\partial \Omega} \Delta \xi_{*} \frac{\partial \xi_{*}}{\partial \nu}
\end{aligned}
$$

where $\xi_{*}$ is the solution of the linear problem

$$
\left\{\begin{array}{l}
-\Delta^{2} \xi_{*}+\Delta \xi_{*}=2 \pi \sum_{i=1}^{d} \delta_{b_{i}} \quad \text { in } \quad \Omega \\
\xi_{*}=0 \quad \text { on } \quad \partial \Omega \\
\frac{\partial}{\partial \nu} \Delta \xi_{*}=-g \quad \text { on } \quad \partial \Omega,
\end{array}\right.
$$

and the function $R$ is given by

$$
R(x)=\Phi(x)-\sum_{i=1}^{d} \log \left|x-b_{i}\right|
$$

where $\Phi$ is the solution of

$$
\left\{\begin{array}{l}
\Delta \Phi=2 \pi \sum_{i=1}^{d} \delta_{b_{i}} \quad \text { in } \quad \Omega \\
\frac{\partial \Phi}{\partial \nu}=g+\frac{\partial \xi}{\partial \nu} \quad \text { on } \quad \partial \Omega
\end{array}\right.
$$

Vol. $12, \mathrm{n}^{\circ} 3-1995$. 
We prove

THEOREM 4. - Let $a_{i}$ be as in theorem 3. The configuration $\left(a_{i}\right)$ minimizes $W$.

The arguments in the proofs of theorems 3 and 4 follow closely the strategy of [2]. Let us however emphasize two important differences. First, we have to use a local version of Pohozaev's identity (by local we mean on balls of radius $\epsilon^{\alpha}$, for $\alpha<1$ ), thereas this identity yielded directly an important estimate in [2]. Second, most of our estimates can be derived from the equation verified by $h$, the magnetic field.

This paper is organized as follows. The next section is devoted to the proof of theorem $\mathbf{l}$ and 2 , and to some general remarks. Section II is devoted to some basic properties of $\left(u_{\epsilon}, A_{\epsilon}\right)$, in particular the GinzburgLandau equations, the maximum principle for $\left|u_{\epsilon}\right|$, and an uniform bound for $\nabla_{A} u$. Section III is devoted to local estimates. Section IV contains a localization of the singularities (in the spirit of [2]). In Section V we deduce global $L^{p}$ estimates for $p<2$. Section VI is devoted to the proof of the convergence. In section VII we derive the London equation verified by $h_{*}$ and we characterize $u_{*}$. Finally Section VIII is devoted to the proof of theorem 4 .

Remarks on Physics. - In the theory of superconductivity, conducting electrons are described as a fluid existing in two phases, the superconducting one and the normal one. In the superconducting state the material has an infinite electric conductivity, and repeals magnetic fields (the London effect). On a microscopic scale, the superconducting state corresponds to a pairing of the electrons. The pairs of electrons are described on a macroscopic scale by a complex-valued function $u$, often called condensate wave function. Roughly speaking, one could think of $|u(x)|$ as representing the density of pairs of superconducting electrons. The Ginzburg-Landau functional then governs the interaction between $u$ and the magnetic field. The parameter $\kappa=\epsilon^{-1}$ is called the Ginzburg-Landau parameter, and depends on the material. Material with different values of $\kappa$ have very different properties.

If $\kappa<\frac{1}{\sqrt{2}}$, the material is called a type I superconductor. If one applies an exterior magnetic field to the sample, then for a critical value of the applied field, the sample passes from the superconducting state to the normal state. For $\kappa \geqslant \frac{1}{\sqrt{2}}$, the situation is quite different and the sample passes gradually from the superconducting to the normal state. This corresponds to the coexistence of the normal and superconducting state: the normal 
phase is confined in vortices or filaments which are quite similar to the ones studied in this paper. for a detailed description see [7], [9], [12] and [16]. We will turn to a mathematical description of the physical situation in a forthcoming paper.

For abelian Higgs models we refer to [11]. See also related problems studied in [8], [13] and [14].

Remarks on notation. $-d$ and $\star$ denote respectively the exterior derivative and the linear operator on the $\mathbb{R}$-valued forms of $\mathbb{R}^{2}$ such that

$\star d x_{1}=d x_{2}, \star d x_{2}=-d x_{1}, \star 1=d x_{1} \wedge d x_{2} \quad$ and $\quad \star d x_{1} \wedge d x_{2}=1$

By $d^{*}$ we mean the operator $\star^{-1} d \star$, where $\star^{-1}$ is the inverse of $\star$.

The scalar product on $\mathbb{C}$ is denoted by $($,$) , i.e. (a, b)=\frac{1}{2}(a \bar{b}+\bar{a} b)$. More generally, if $f=f_{I} d x^{I}$ and $g=g_{J} d x^{J}$ are two forms with coefficients in $\widetilde{C},(f \wedge g)$ denotes the 2l-form with real coefficients $\sum\left(f_{I}, g_{J}\right) d x^{I} \wedge d x^{J}$.

$\times$ represents the wedge product between two vectors of $I^{2}$, it is considered as a real number.

We often identify a 1-form and the associated vector by the scalar product. We also often identify a vector of $\mathbb{R}^{2}$ and the corresponding complex number. For instance, as a result of our identifications, $u \times \frac{\partial u}{\partial \tau}$ stands for $\left(i u, \frac{\partial u}{\partial \tau}\right)$

\section{THE VARIATIONAL PROBLEM}

We begin this section by some definitions and remarks.

Definition I.1. - We say that $(u, A) \in H^{1}\left(\Omega, R^{2}\right) \times H^{1}\left(\Omega, \mathbb{R}^{2}\right)$ is gauge equivalent to $(v, B) \in H^{1}\left(\Omega, R^{2}\right) \times H^{1}\left(\Omega, R^{2}\right)$, if there exists a function $\phi \in H^{2}(\Omega)$ such that

$$
\left\{\begin{array}{l}
v=e^{i \phi} u \\
B=A+d \phi
\end{array}\right.
$$

As we have mentionned in the introduction, it is often very useful to reduce the gauge invariance of the problem by imposing a condition on $A$. We have the standard result: 
Proposition I.1. - Let $(u, A) \in H^{1}(\Omega) \times H^{1}(\Omega)$. There is some $(\tilde{u}, \tilde{A}) \in H^{1}(\Omega) \times H^{1}(\Omega)$, gauge equivalent to $(u, A)$ such that

$$
\left\{\begin{array}{lll}
d^{*} \tilde{A}=0 & \text { in } & \Omega \\
\tilde{A} . \nu=0 & \text { on } & \partial \Omega
\end{array}\right.
$$

Remark. - (I.1) is often termed a Coulomb or Lorentz gauge.

Proof. - Consider the linear problem

$$
\left\{\begin{array}{lll}
\Delta \phi=-d^{*} A & \text { in } & \Omega \\
\frac{\partial \phi}{\partial \nu}=-A . \nu & \text { on } & \partial \Omega .
\end{array}\right.
$$

We easily verify that

$$
\left\{\begin{array}{l}
\tilde{u}=e^{i \phi} u \\
\tilde{A}=A+d \phi
\end{array}\right.
$$

satisfies (I.1).

In the course of our proof, we will often need local versions of proposition I.1. We have

Proposition I.2. - Let $G$ be some smooth subdomain of $\Omega$, and let $(u, A)$ be smooth. There is some smooth $(\tilde{u}, \tilde{A})$ gauge equivalent to $(u, A)$ such that

$$
\left\{\begin{array}{lll}
d^{*} \tilde{A}=0 & \text { in } & G \\
\tilde{A} \cdot \nu=0 & \text { on } & \partial G .
\end{array}\right.
$$

Note that, in proposition I.2, we impose only a condition for $\tilde{A}$ in $G$ and on $\partial G$. The proof is the same as the proof of proposition I.1 (performed in $\mathrm{G})$, except that we have to extend $\phi$ to $\Omega$ in a smooth way.

Proof of theorem $I$ and 2 . - Let $\left(u_{n}, A_{n}\right)$ be a minimizing sequence for (8). In view of proposition I.I we may assume that

$$
\left\{\begin{array}{lll}
d^{*} A_{n}=0 & \text { in } & \Omega \\
A_{n} \cdot \nu=0 & \text { on } & \partial \Omega .
\end{array}\right.
$$

We deduce from (I.7) that there is some $\xi_{n} \in H^{2}(\Omega)$ such that $\left(A_{n}^{1}, A_{n}^{2}\right)=\left(-\xi_{n_{x_{2}}}, \xi_{n_{x_{1}}}\right)$, where $\left(x_{1}, x_{2}\right)$ are cartesian coordinates on $I^{2}$. Hence

$$
\begin{cases}\Delta \xi_{n}=\star d A_{n} & \text { in } \\ \xi_{n}=0 & \Omega\end{cases}
$$


It follows, by standard elliptic estimates, that

$$
\begin{aligned}
\int_{\Omega}\left|A_{n}\right|^{2}+\int_{\Omega}\left|\nabla A_{n}\right|^{2} & =\int_{\Omega}\left|\nabla \xi_{n}\right|^{2}+\int_{\Omega}\left|\nabla^{2} \xi_{n}\right|^{2} \\
& \leqslant\left|\xi_{n}\right|_{W^{2,2}} \leqslant \int_{\Omega}\left|d A_{n}\right|^{2} \\
& \leqslant C G_{\epsilon}\left(u_{n}, A_{n}\right)<C .
\end{aligned}
$$

Hence $A_{n}$ is uniformly bounded in $H^{1}(\Omega)$. Since

$$
\int_{\Omega}\left|\nabla u_{n}-i A_{n} u_{n}\right|^{2} \leqslant 2 G_{\epsilon}\left(u_{n}, A_{n}\right)<C,
$$

we obtain

$$
\begin{aligned}
\int_{\Omega}\left|\nabla u_{n}\right|^{2} & \leqslant C+2 \int_{\Omega} A_{n}^{2}\left|u_{n}\right|^{2} \leqslant C+2 \int_{\Omega} A_{n}^{2}\left(1-\left|u_{n}\right|^{2}\right)+2 \int_{\Omega} A_{n}^{2} \\
& \leqslant C\left(\int_{\Omega} A_{n}^{4}\right)^{\frac{1}{2}} \leqslant C .
\end{aligned}
$$

We deduce that $u_{n}$ is also a bounded sequence in $H^{1}\left(\Omega, R^{2}\right)$. Passing to a subsequence if necessary we have

$$
\begin{array}{ccc}
u_{n}-u \quad \text { in } & H^{1}(\Omega) \text { weakly } \\
A_{n} \rightarrow A & \text { in } \quad H^{1}(\Omega) \text { weakly. }
\end{array}
$$

Passing to the limit in (I.7) we are led to

$$
\left\{\begin{array}{lll}
d^{*} A=0 & \text { in } & \Omega \\
A . \nu=0 & \text { on } & \partial \Omega .
\end{array}\right.
$$

Moreover by lower semicontinuity

$$
\begin{aligned}
G_{\epsilon}(u, A) & \leqslant \liminf _{n \rightarrow+\infty} G_{\epsilon}\left(u_{n}, A_{n}\right) \\
& \leqslant \operatorname{Inf}_{(v, B) \in V} G_{\epsilon}(v, B) .
\end{aligned}
$$

It remains to prove that $(u, A) \in V$, that is that the boundary conditions are preserved.

We clearly have

$$
A_{n} . \tau \rightarrow A . \tau \quad \text { in } \quad H^{1 / 2}(\partial \Omega)
$$

Vol. $12, \mathrm{n}^{\circ} 3-1995$. 
On the other hand, we deduce from the condition $J_{n} \cdot \tau=g$ that

$$
\frac{\partial u_{n}}{\partial \tau}=i u_{n}\left(g+A_{n} \cdot \tau\right) \quad \text { on } \quad \partial \Omega
$$

Since by (I.9) $u_{n} \rightarrow u$ in $H^{1 / 2}(\partial \Omega)$ weakly, we see that $u_{n} \rightarrow u$ strongly in $L^{p}(\partial \Omega)$ for any $1<p<+\infty$. Similarly we may infer that $g+A_{n} . \tau \rightarrow g+A . \tau$ strongly in $L^{p}(\partial \Omega)$. It follows that

$\frac{\partial u_{n}}{\partial \tau} \rightarrow i u(g+A . \tau)$ strongly in $L^{p}(\partial \Omega)$ for any $1<p<+\infty$

Passing to the limit (in the sense of distributions) in (I.15) we obtain

$$
\frac{\partial u}{\partial \tau}=i u(g+A . \tau) \quad \text { on } \quad \partial \Omega
$$

This leads to

$$
J . \tau=\left(i u, \tau . \nabla_{A} u\right)=g .
$$

(where $\nabla_{A} u=\nabla u-i A u$ ).

Finally it remains to prove that

$$
\operatorname{deg}(u, \partial \Omega)=d
$$

We have

$$
\operatorname{deg}(u, \partial \Omega)=\frac{1}{2 \pi} \int_{\partial \Omega} u \times \frac{\partial u}{\partial \tau}=\frac{1}{2 \pi} \int_{\partial \Omega}(g+A . \tau)
$$

and

$$
d=\operatorname{deg}\left(u_{n}, \partial \Omega\right)=\frac{1}{2 \pi} \int_{\partial \Omega}\left(g+A_{n} \cdot \tau\right) \longrightarrow \frac{1}{2 \pi} \int_{\partial \Omega}(g+A . \tau)
$$

by (I.14), and (I.19) follows. This completes the proof of theorem 1. Since $A$ satisfies (I.11), we also have proved theorem 2 . 


\section{BASIC PROPERTIES OF $\left(u_{\epsilon}, A_{\epsilon}\right)$}

\subsection{The Ginzburg-Landau equation}

Proposition II.1. - Any minimizer $\left(u_{\epsilon}, A_{\epsilon}\right)$ of $G_{\epsilon}$ on $V$ satisfies the Ginzburg-Landau equations on $\Omega$

$$
\begin{gathered}
-\nabla_{A_{\epsilon}}^{2} u_{\epsilon}=\frac{1}{\epsilon^{2}} u_{\epsilon}\left(1-\left|u_{\epsilon}\right|^{2}\right) \\
-\star d h_{\epsilon}=J_{\epsilon}
\end{gathered}
$$

where $J_{\epsilon}=\left(i u_{\epsilon}, d_{A_{\epsilon}} u_{\epsilon}\right)$, and $d_{A_{\epsilon}} u_{\epsilon}=d u_{\epsilon}-i A_{\epsilon} u_{\epsilon}$.

For a proof see for instance [11], [9]. Remark that equations (II.1) and (II.2) are independent of the gauge (in particular they hold even if we do not assume (9)). We recall that $\nabla_{A}^{2}$ is the operator defined by

$$
\nabla_{A}^{2}=\sum_{j=1}^{2}\left(\frac{\partial}{\partial x_{j}}-i A_{j}\right)\left(\frac{\partial}{\partial x_{j}}-i A_{j}\right) .
$$

Recall also that, in view on our variational problem, equations (II.1) and (II.2) are completed with the boundary conditions

$$
\begin{array}{lll}
J_{\epsilon}, \tau=g & \text { on } & \partial \Omega \\
\left|u_{\epsilon}\right|=1 & \text { on } & \partial \Omega .
\end{array}
$$

Moreover we have chosen $\left(u_{\epsilon}, A_{\epsilon}\right)$ so that (I.1) is satisfied.

Proposition II.2. - Any minimizer $\left(u_{\epsilon}, A_{\epsilon}\right)$ to $(8)$ in $V$ satisfies

$$
\left|u_{\epsilon}\right| \leqslant 1 \quad \text { in } \Omega .
$$

Moreover if $A_{\epsilon}$ satisfies (1.1), then $\left(u_{\epsilon}, A_{\epsilon}\right)$ are smooth.

Proof. - See [11], [7] for the proof of regularity of $\left(u_{\epsilon}, A_{\epsilon}\right)$. Inequality (II.5) is a consequence of the maximum principle, since we have

$$
\frac{1}{2} \Delta\left|u_{\epsilon}\right|^{2}=-\frac{1}{\epsilon^{2}}\left|u_{\epsilon}\right|^{2}\left(1-\left|u_{\epsilon}\right|^{2}\right)+\left|\nabla_{A_{\epsilon}} u_{\epsilon}\right|^{2} .
$$

A very useful consequence of propositions II. 1 and II.2 is the following. Proposition II.3. - We have

$$
\frac{\partial h_{\epsilon}}{\partial \nu}=-g \quad \text { on } \quad \partial \Omega .
$$


Proof. - Since $h_{\varepsilon}$ is smooth up to $\partial \Omega$, (II.2) holds on $\partial \Omega$ and yields (II.7).

The next equality plays the same role as Theorem III.3 in [2] and is a Pohozaev type equality.

Proposition II.4. - Let $G$ be a subdomain of $\Omega$. We have

$$
\begin{aligned}
\int_{G} \frac{1}{\epsilon^{2}}\left(1-\left|u_{\epsilon}\right|^{2}\right)^{2}-h_{\epsilon}^{2}= & \int_{\partial G}(x . \nu)\left[\left(\tau . \nabla_{A_{\epsilon}} u_{\epsilon}\right)^{2}-\left(\nu . \nabla_{A_{\epsilon}} u_{\epsilon}\right)^{2}\right] \\
& +\int_{\partial G} \frac{1}{2}(x . \nu)\left(\frac{1}{\epsilon^{2}}\left(1-\left|u_{\epsilon}\right|^{2}\right)^{2}-h_{\epsilon}^{2}\right) \\
& -\int_{\partial G}(x . \tau)\left(\tau . \nabla_{A_{\epsilon}} u_{\epsilon}, \nu . \nabla_{A_{\epsilon}} u_{\epsilon}\right) .
\end{aligned}
$$

Proof. - Use the stress - energy tensor as defined in [11] and integrate by parts on $G$.

\subsection{An upper bound for the energy of minimizers}

As in [2] (theorem III.1), we may derive the following upper bound.

Proposition II.5. - We have, for $\epsilon<1$

$$
G_{\epsilon}\left(u_{\epsilon}, A_{\epsilon}\right) \leqslant \pi d \log \frac{1}{\epsilon}+C
$$

where $C$ depends only on $\Omega, g$ and $d$.

Remark. - The proposition below, as written, could have been a direct consequence of Theorem III.1 of [2], but here we establish a specific dependence of $C$ on $\Omega, g$ and $d$ : for a given degree $d$ we prove that $C$ is independent of the scale of $\Omega$, by changing $\Omega$ into $\Omega_{\lambda}=\frac{1}{\lambda} \Omega$, and replacing $g$ by $g_{\lambda}(x)=\lambda g(\lambda x)$ for $\lambda<1$. This scale invariance, that we will use during the proof of proposition II.6, represents one of the major difference between the gauge invariant functional, $G$, and the non gauge invariant one, $F$. One of its consequence is illustrated for instance by the fact that we may consider the functional $G$ on all of $\mathbb{R}^{2}$. Even if we prescribe some (non-zero) degree at infinity the functional may remain bounded (see [11]). This is not the case for the functional $F$ (see [5]). 
VORTICES FOR A VARIATIONAL PROBLEM RELATED TO SUPERCONDUCTIVITY 255

Proof. - It suffices to construct an explicit test function $\left(v_{\epsilon}, B_{\epsilon}\right) \in V$ such that

$$
G_{\epsilon}\left(v_{\epsilon}, B_{\epsilon}\right) \leqslant \pi d \log \frac{1}{\epsilon}+C .
$$

To that aim, we proceed as in [2] and fix $d$ distinct points $a_{1}, \ldots, a_{d}$ in $\Omega$, and $R>0$ so small that

$$
B\left(a_{i}, R\right) \subset \Omega
$$

and

$$
B\left(a_{i}, R\right) \cap B\left(a_{j}, R\right)=\emptyset \quad \text { if } i \neq j
$$

Set

$$
\tilde{\Omega}=\Omega \backslash \bigcup_{i=1}^{d} B\left(a_{i}, R\right),
$$

and let $w_{0}$ be a smooth map from $\tilde{\Omega}$ to $S^{1}$ verifying for all $j$

$$
w_{0}=e^{i \theta}=\frac{z-a_{j}}{\left|z-a_{j}\right|} \quad \text { on } \quad \partial B\left(a_{j}, R\right)
$$

Since $w_{0}$ is a smooth map from $\tilde{\Omega}$ to $S^{1}$ we have

$$
2 \frac{\partial w_{0}}{\partial x_{1}} \times \frac{\partial w_{0}}{\partial x_{2}}=\frac{\partial}{\partial x_{1}}\left(w_{0} \times \frac{\partial w_{0}}{\partial x_{2}}\right)-\frac{\partial}{\partial x_{2}}\left(w_{0} \times \frac{\partial w_{0}}{\partial x_{1}}\right)=0
$$

We set

$$
\begin{aligned}
& B_{1}^{0}=w_{0} \times \frac{\partial w_{0}}{\partial x_{1}}=\left(i w_{0}, \frac{\partial w_{0}}{\partial x_{1}}\right) \\
& B_{2}^{0}=w_{0} \times \frac{\partial w_{0}}{\partial x_{2}}=\left(i w_{0}, \frac{\partial w_{0}}{\partial x_{2}}\right)
\end{aligned}
$$

We verify easily that

$$
\left(\nabla-i B^{0}\right) w_{0}=0 \quad \text { in } \quad \tilde{\Omega}
$$

and

$$
d B^{0}=0 \quad \text { in } \quad \tilde{\Omega} .
$$

Let $B^{1}$ be the solution of

$$
\left\{\begin{array}{l}
\Delta B^{1}=0 \quad \text { in } \quad \Omega \\
B^{1} \cdot \tau=g, \quad B^{1} \cdot \nu=0 \quad \text { on } \quad \partial \Omega
\end{array}\right.
$$

Vol. 12, n 3-1995. 
We deduce from elliptic estimates that

$$
\int_{\Omega}\left|B^{1}\right|^{2}+\left|\nabla B^{1}\right|^{2}<C .
$$

On $\tilde{\Omega}$ we set

$$
B_{\epsilon}=B^{0}+B^{1} \quad \text { and } \quad v_{\epsilon}=w_{0}
$$

we verify that

$$
\begin{gathered}
\left|v_{\epsilon}\right|=1 \quad \text { in } \tilde{\Omega} \\
\left(\nabla-i B_{\epsilon}\right) v_{\epsilon}=-i B^{1} v_{\epsilon} \quad \text { in } \tilde{\Omega}
\end{gathered}
$$

and that

$$
d B_{\varepsilon}=d B^{1} \quad \text { in } \quad \tilde{\Omega} .
$$

Hence we compute

$$
\begin{aligned}
& \frac{1}{2} \int_{\tilde{\Omega}}\left|\left(\nabla-i B_{\epsilon}\right) v_{\epsilon}\right|^{2}+\left|d B_{\epsilon}\right|^{2}+\frac{1}{2 \epsilon^{2}}\left(1-\left|v_{\epsilon}\right|^{2}\right)^{2} \\
& \quad=\frac{1}{2} \int_{\tilde{\Omega}}\left|B^{1}\right|^{2}+\left|d B^{1}\right|^{2}<C .
\end{aligned}
$$

In view of (II.13).

It remains to define $\left(v_{\epsilon}, B_{\epsilon}\right)$ on $\bigcup_{i=1}^{d} B\left(a_{i}, R\right)$. Let $\zeta$ be a smooth function from $R$ to $\mathbb{R}^{+}$such that

$$
\begin{array}{ll}
\zeta(t)=1 & \text { if } t \geqslant 1 \\
\zeta(t)=0 & \text { if } t \leqslant \frac{1}{2} .
\end{array}
$$

We set, on $B\left(a_{i}, R\right)$

$$
v_{\epsilon}(x)=\frac{x-a_{i}}{\left|x-a_{i}\right|} \zeta\left(\frac{x-a_{i}}{\epsilon}\right), \quad \text { as soon as } \quad \epsilon<R,
$$

and

$$
B_{\epsilon}(x)=B_{\epsilon}\left(\frac{x-a_{i}}{\left|x-a_{i}\right|} R\right) \zeta\left(\frac{x-a_{i}}{R}\right)
$$


A simple computation yields

$$
\begin{aligned}
& \frac{1}{2} \int_{B\left(a_{i}, R\right)}\left|\left(\nabla-i B_{\epsilon}\right) v_{\epsilon}\right|^{2}+\left|d B_{\epsilon}\right|^{2} \\
& \quad+\frac{1}{2 \epsilon^{2}}\left(1-\left|v_{\epsilon}\right|^{2}\right)^{2}<\pi \log \left(\frac{1}{\epsilon}\right)+C
\end{aligned}
$$

where $C$ is a constant depending only on $R$. Clearly we have $\left(v_{\epsilon}, B_{\epsilon}\right) \in$ $H^{1}(\Omega) \times H^{1}(\Omega)$ and combining (II.14) and (II.15) we obtain

$$
G_{\epsilon}\left(v_{\epsilon}, B_{\epsilon}\right) \leqslant \pi d \log \frac{1}{\epsilon}+C .
$$

On the other hand we verify that

$$
\begin{gathered}
\operatorname{deg}\left(v_{\epsilon}, \partial \Omega\right)=\operatorname{deg}\left(w_{0}, \partial \Omega\right)=d \\
\left|v_{\epsilon}\right|=\left|w_{0}\right|=1 \quad \text { on } \quad \partial \Omega
\end{gathered}
$$

and

$$
J_{\epsilon} \cdot \tau=B^{1} \cdot \tau=g \quad \text { on } \quad \partial \Omega .
$$

Hence $\left(v_{\epsilon}, B_{\epsilon}\right) \in V$, and (II.9) follows from (II.16).

\subsection{An estimate for $\left|\nabla_{A_{\epsilon}} u_{\epsilon}\right|$}

The equivalent of lemma $A 2$ of [1] is the following.

Proposition II.6. - We have

$$
\left|\nabla_{A_{\epsilon}} u_{\epsilon}\right| \leqslant C / \epsilon,
$$

and hence

$$
\left|\nabla h_{\epsilon}\right| \leqslant\left|\nabla_{A_{\epsilon}} u_{\epsilon}\right| \leqslant C / \epsilon,
$$

where $C$ is some constant depending only on $g, \Omega$ and $d$.

Proof. - We deduce from proposition II.5 that

$$
\int_{\Omega}\left|h_{\epsilon}\right|^{2} \leqslant C \log \frac{1}{\epsilon} .
$$

It will be convenient to make the following change of scale. Set

$$
\bar{x}=\frac{x}{\epsilon} \quad \text { and } \quad \bar{\Omega}=\frac{1}{\epsilon} \Omega,
$$

Vol. $12, n^{\circ} 3-1995$. 


$$
\bar{u}(\bar{x})=u_{\epsilon}(\epsilon \bar{x})=u_{\epsilon}(x) \quad \text { i.e } \quad u_{\epsilon}(x)=\bar{u}(x / \epsilon)
$$

and

$$
\bar{A}(\bar{x})=\epsilon A_{\epsilon}(\epsilon \bar{x}) .
$$

Hence

$$
\nabla_{A} \bar{u}(\bar{x})=\epsilon \nabla_{A_{\epsilon}} u_{\epsilon}(\epsilon \bar{x})=\epsilon \nabla_{A_{\epsilon}} u_{\epsilon}(x),
$$

and

$$
\bar{h}(\bar{x})=\star d \bar{A}(\bar{x})=\epsilon^{2} h_{\epsilon}(\epsilon \bar{x})=\epsilon^{2} h_{\epsilon}(x) .
$$

It follows that $(\bar{u}, \bar{A})$ is a minimizer of the functional

$$
\bar{G}(v, B)=\int_{\bar{\Omega}} \frac{1}{2}|(\nabla-i B) v|^{2}+\frac{1}{2 \epsilon^{2}}|d B|^{2}+\frac{1}{4}\left(1-|v|^{2}\right)^{2}
$$

on the space

$$
\bar{V}=\left\{\begin{array}{c}
(v, B) \in H^{1}(\bar{\Omega}) \times H^{1}(\bar{\Omega}) \text { such that } \\
|v|=1 \text { on } \partial \bar{\Omega} \\
J . \tau=\bar{g} \operatorname{deg}(v, \partial \bar{\Omega})=d
\end{array}\right\}
$$

where $\bar{g}(\bar{x})=\epsilon g(\epsilon \bar{x})$.

The Ginzburg-Landau equations for $(\bar{u}, \bar{A})$ are therefore

$$
\begin{aligned}
& -\nabla_{\bar{A}}^{2} \bar{u}=\bar{u}\left(1-|\bar{u}|^{2}\right) \quad \text { in } \bar{\Omega} \\
& \frac{1}{\epsilon^{2}} d^{*} d \bar{A}=\left(i \bar{u}, d_{\bar{A}} \bar{u}\right) .
\end{aligned}
$$

since $d^{*} \bar{A}=0$, (II.25) implies

$$
-\frac{1}{\epsilon^{2}} \Delta \bar{A}+|\bar{u}|^{2} \bar{A}=(-i \bar{u}, d \bar{u}) \quad \text { in } \bar{\Omega} .
$$

Let $\bar{x}_{0} \in \bar{\Omega}$, and consider the ball $B\left(\bar{x}_{0}, 2\right)$. We are going to show that

$$
\left|\nabla_{\bar{A}} \bar{u}\right| \leqslant C \quad \text { in } \quad B\left(\bar{x}_{0}, 1\right) .
$$

To that aim we distinguish two cases.

Case 1. $-\operatorname{dist}\left(\bar{x}_{0}, \partial \Omega\right)>2$. It follows that $B\left(\bar{x}_{0}, 2\right) \cap \partial \bar{\Omega}=\emptyset$. In view of proposition I.2 we may choose $(\tilde{u}, \tilde{A})$ gauge equivalent to $(\bar{u}, \bar{A})$ such that

$$
\left\{\begin{array}{lll}
d^{*} \tilde{A}=0 & \text { in } & B\left(\bar{x}_{0}, 2\right) \\
\tilde{A} \cdot \nu=0 & \text { on } & \partial B\left(\bar{x}_{0}, 2\right) .
\end{array}\right.
$$


Hence there is some $\tilde{\xi} \in H^{2}\left(B\left(\bar{x}_{0}, 2\right)\right)$ such that

$$
\tilde{A}=\left(-\tilde{\xi}_{x_{2}}, \tilde{\xi}_{x_{1}}\right),
$$

where $\tilde{\xi}$ satisfies

$$
\begin{cases}\Delta \tilde{\xi}=\bar{h} & \text { in } \quad B\left(\bar{x}_{0}, 2\right) \\ \tilde{\xi}=0 & \text { on } \quad \partial B\left(\bar{x}_{0}, 2\right) .\end{cases}
$$

By standard elliptic estimates, we obtain

$$
\|\nabla \xi\|_{L^{2}\left(B\left(\bar{x}_{0}, 2\right)\right)}+\left\|\nabla^{2} \xi\right\|_{L^{2}\left(B\left(\bar{x}_{0}, 2\right)\right)} \leqslant C\|\bar{h}\|_{L^{2}(\bar{\Omega})} \leqslant C \epsilon|\log \epsilon|^{1 / 2},
$$

where we have used (II.19) and (II.24). It follows that

$$
\|\tilde{A}\|_{H^{1}\left(B\left(\bar{x}_{0}, 2\right)\right)}^{2} \leqslant C \epsilon^{2} \log \epsilon .
$$

In the gauge $\tilde{A}$, (II.25) becomes

$$
-\Delta \tilde{u}=\tilde{u}\left(1-|\tilde{u}|^{2}\right)-\tilde{A}^{2} \tilde{u}-2 i \tilde{A} \cdot d \tilde{u} .
$$

Where $\tilde{A} \cdot d \tilde{u}=\tilde{A}_{1} \tilde{u}_{x_{1}}+\tilde{A}_{2} \tilde{u}_{x_{2}}$. We write

$$
\tilde{u}=\tilde{u}_{0}+\tilde{u}_{1} \quad \text { on } \quad B\left(\bar{x}_{0}, 2\right),
$$

where $\tilde{u}_{0}$ satisfies

$$
\left\{\begin{array}{l}
\Delta \tilde{u}_{0}=0 \quad \text { in } \quad B\left(\bar{x}_{0}, 2\right) \\
\tilde{u}_{0}=\tilde{u} \quad \text { on } \quad \partial B\left(\bar{x}_{0}, 2\right),
\end{array}\right.
$$

and $\tilde{u}_{1}$ satisfies

$$
\left\{\begin{array}{l}
-\Delta \tilde{u}_{1}=\tilde{u}\left(1-|\tilde{u}|^{2}\right)-\tilde{A}^{2} \tilde{u}-2 i \tilde{A} . d \tilde{u} \quad \text { in } B\left(\bar{x}_{0}, 2\right) \\
\tilde{u}_{1}=0 \quad \text { on } \partial B\left(\bar{x}_{0}, 2\right) .
\end{array}\right.
$$

From elliptic estimates, we obtain

$$
\left|\nabla \tilde{u}_{0}\right|<C \quad \text { on } \quad B\left(\bar{x}_{0}, 3 / 2\right) .
$$

We have for any $p<+\infty$, by (II.29)

$$
\left\|\tilde{A}^{2} \tilde{u}\right\|_{L^{p}} \leqslant\left\|\tilde{A}^{2}\right\|_{L^{p}}=\|\tilde{A}\|_{L^{2 p}}^{2} \leqslant\|\tilde{A}\|_{H^{1}}^{2} \leqslant C \epsilon^{2}|\log \epsilon|
$$

Vol. $12, \mathrm{n}^{\circ} 3-1995$. 
and for $p<2$,

$$
\begin{aligned}
\|\tilde{A} . \nabla \tilde{u}\|_{L^{p}} & \leqslant\left\|\tilde{A} . \nabla_{\tilde{A}} \tilde{u}\right\|_{L^{p}}+\left\|\tilde{A}^{2} \tilde{u}\right\|_{L^{p}} \\
& \leqslant\left\|\nabla_{\tilde{A}} \tilde{u}\right\|_{L^{2}}\|\tilde{A}\|_{L^{p q}}+\left\|\tilde{A}^{2}\right\|_{L^{p}},
\end{aligned}
$$

where $\frac{1}{q}+\frac{p}{2}=1$. Hence, since $\| \nabla_{\tilde{A}} \tilde{u}||_{L^{2}(\bar{\Omega})} \leqslant C|\log \epsilon|$ and $\|\tilde{A}\|_{L^{p q}} \leqslant$ $\|\tilde{A}\|_{H^{1}}$, we deduce, by (II.29) that

$$
\| \tilde{A} .\left.\nabla \tilde{u}\right|_{L^{p}} \leqslant C \epsilon|\log \epsilon|^{3 / 2}
$$

and from (II.31), (II.33), and (II.35) that

$$
\left\|\tilde{u}_{1}\right\|_{W^{2, p}\left(B\left(\bar{x}_{0}, 2\right)\right)} \leqslant C_{p} \epsilon|\log \epsilon|^{3 / 2} \quad \forall p<2 .
$$

Hence, by Sobolev imbedding

$$
\left\|\nabla \tilde{u}_{1}\right\|_{L^{p}\left(B\left(\bar{x}_{0}, 2\right)\right)} \leqslant C_{p} \epsilon|\log \epsilon|^{3 / 2} \quad \forall 1<p<+\infty .
$$

Combining (II.36) and (II.32) we are led to

$$
\|\nabla \tilde{u}\|_{L^{p}\left(B\left(\bar{x}_{0}, 3 / 2\right)\right)} \leqslant C_{p} \quad \forall 1<p<+\infty
$$

Working now on the ball $B\left(\bar{x}_{0}, 3 / 2\right)$ instead of the ball $B\left(\bar{x}_{0}, 2\right)$, we see that equation (II.31) leads to

$$
\left\|\tilde{u}_{1}\right\|_{W^{2, P}\left(B\left(\bar{x}_{0}, 3 / 2\right)\right)} \leqslant C_{p} \quad \forall 1<p<+\infty .
$$

This yields in particular, by embedding

$$
\left|\nabla \tilde{u}_{1}\right| \leqslant C \quad \text { on } \quad B\left(\bar{x}_{0}, 3 / 2\right)
$$

which shows, combining with (II.32) that

$$
|\nabla \tilde{u}| \leqslant C \quad \text { on } \quad B\left(\bar{x}_{0}, 3 / 2\right) \text {. }
$$

Using next equation (II.26) on $B\left(\bar{x}_{0}, 3 / 2\right)$ we deduce that

$$
\|\tilde{A}\|_{L^{\infty}\left(B\left(\bar{x}_{0}, 1\right)\right)} \leqslant C .
$$

Combining (II.38) and (II.39) we deduce (II.27) in case 1. 
Case 2. $-\bar{x}_{0} \in \partial \bar{\Omega}$. We follow essentially the same arguments. we choose $(\tilde{u}, \tilde{A})$ gauge equivalent to $(\bar{u}, \bar{A})$ such that

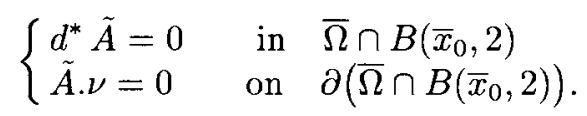

As above, we write

$$
\tilde{u}=\tilde{u}_{0}+\tilde{u}_{1},
$$

where $\tilde{u}_{0}$ verifies

$$
\left\{\begin{array}{l}
\Delta \tilde{u}_{0}=0 \quad \text { on } \quad \bar{\Omega} \cap B\left(\bar{x}_{0}, 2\right) \\
\tilde{u}_{0}=\tilde{u} \quad \text { on } \quad \partial\left(\bar{\Omega} \cap B\left(\bar{x}_{0}, 2\right) .\right.
\end{array}\right.
$$

We have $\tilde{A}=\left(-\tilde{\xi}_{x_{2}}, \tilde{\xi}_{x_{1}}\right)$ where $\tilde{\xi}$ satifies

$$
\left\{\begin{array}{l}
\Delta \tilde{\xi}=\bar{h} \quad \text { on } \quad \bar{\Omega} \cap B\left(\bar{x}_{0}, 2\right) \\
\tilde{\xi}=0 \quad \text { on } \quad \partial\left(\bar{\Omega} \cap B\left(\bar{x}_{0}, 2\right)\right) .
\end{array}\right.
$$

We have

$$
\tilde{A} . \tau=\frac{\partial \tilde{\xi}}{\partial \nu}
$$

and

$$
\frac{\partial \tilde{u}}{\partial \tau}=i\left(\frac{\partial \tilde{\xi}}{\partial \nu}+g\right) \tilde{u} \quad \text { on } \quad \partial \bar{\Omega} \cap B\left(\bar{x}_{0}, 2\right) .
$$

From (II.40) and (II.42) we deduce, by classical linear estimates, that

$$
\left.\left\|\frac{\partial \tilde{u}}{\partial \tau}\right\|\right|_{H^{1 / 2}\left(\partial \bar{\Omega} \cap B\left(\bar{x}_{0}, 3 / 2\right)\right)} \leqslant C .
$$

Hence since $\tilde{u}_{0}$ is harmonic we deduce that

$$
\left\|\tilde{u}_{0}\right\|_{H^{2}\left(B\left(\bar{x}_{0}, 3 / 2\right) \cap \Omega\right)} \leqslant C .
$$

As for (II.29) we have

$$
\|\tilde{A}\|_{H^{1}\left(B\left(\bar{x}_{0}, 3 / 2\right) \cap \Omega\right)} \leqslant C .
$$

Similarly arguing as in (II.31)-(II.38), we derive

$$
\|\tilde{u}\|_{H^{1}\left(B\left(\bar{x}_{0}, 3 / 2\right) \cap \bar{\Omega}\right)} \leqslant C .
$$

Vol. 12, $n^{\circ} 3-1995$. 
In particular, we obtain

$$
\left\|\nabla_{\tilde{A}} \tilde{u}\right\|_{H^{1}\left(B\left(\bar{x}_{0}, 3 / 2\right) \cap \bar{\Omega}\right)} \leqslant C .
$$

On the other hand, we have by (II.2)

$$
-\star d \bar{h}=\epsilon^{2}\left(i \tilde{u}, d_{\tilde{A}} \tilde{u}\right)
$$

Hence, we deduce from (II.44), (II.45) and (II.46)

$$
|\nabla \bar{h}|_{L^{2}\left(B\left(\overline{x_{0}}, 3 / 2\right) \cap \bar{\Omega}\right)} \leqslant \epsilon^{2}\left|\nabla_{\tilde{A}} \tilde{u}\right|_{L^{2}\left(B\left(\overline{x_{0}}, 3 / 2\right) \cap \Omega\right)} \leqslant C \epsilon^{2} .
$$

Hence, since $|\bar{h}|_{L^{2}(\bar{\Omega})} \leqslant C \epsilon \log (1 / \epsilon)$, we obtain by Sobolev embedding, for any $p>2$

$$
|\bar{h}|_{L^{p}\left(B\left(\overline{x_{0}}, 3 / 2\right) \cap \Omega\right)} \leqslant C \epsilon^{2} \log \left(\frac{1}{\epsilon}\right) \longrightarrow 0 .
$$

Iterating the argument we deduce that

$$
\left\|\tilde{u}_{0}\right\|_{W^{2, p}\left(B\left(\bar{x}_{0}, 5 / 4\right) \cap \bar{\Omega}\right)} \leqslant C
$$

and hence

$$
\left|\nabla \tilde{u}_{0}\right| \leqslant C
$$

We complete the proof of (II.27) as in case 1.

\section{LOCAL ESTIMATES}

As we may see from proposition II.6, $\epsilon$ is the characteristic length of variations of $\left(u_{\epsilon}, A_{\epsilon}\right)$. The aim of this section is to derive estimates on domains of a larger scale, of order $\epsilon^{\alpha}$ for $\alpha<1$. These estimates will be an important step for deriving global estimates on $\Omega$ (see section $V$ ).

Let $0<\alpha<1$ be fixed. If not otherwise necessary, we drop the indice $\epsilon$ for $u_{\epsilon}, h_{\epsilon}, A_{\epsilon}$ and simply denote $u, h, A$.

Proposition III.1. - Let $0<\alpha<1$ and let $x_{0} \in \Omega$. We have

$$
\int_{B\left(x_{0}, \epsilon^{\alpha}\right) \cap \Omega}|h|^{2} \leqslant C \epsilon^{\alpha}|\log \epsilon|
$$


and

$$
\frac{1}{\epsilon^{2}} \int_{B\left(x_{0}, \epsilon^{\alpha}\right) \cap \Omega}\left(1-|u|^{2}\right)^{2} \leqslant C,
$$

where $C$ depends only on $g, d, \alpha$ and $\Omega$.

Proof. Step 1. Proof of (III.1). - We have, by (II.2),

$$
|\nabla h| \leqslant\left|\nabla_{A} u\right|
$$

and hence by proposition II.5.

$$
\int_{\Omega}|\nabla h|^{2} \leqslant C|\log \epsilon|
$$

On the other hand, we have also

$$
\int_{\Omega}|h|^{2} \leqslant C|\log \epsilon|
$$

Hence, by Sobolev embedding, we deduce that for $p>2$

$$
\left(\int_{\Omega}|h|^{p}\right)^{\frac{1}{p}} \leqslant C_{p}|\log \epsilon|^{\frac{1}{2}}
$$

It follows that

$$
\begin{aligned}
\left(\int_{B\left(x_{0}, \epsilon^{\alpha}\right) \cap \Omega}|h|^{2}\right)^{\frac{1}{2}} & \leqslant\left(\int_{\Omega}|h|^{p}\right)^{\frac{1}{p}}\left[\text { meas }\left(B\left(x_{0}, \epsilon^{\alpha}\right)\right]^{\frac{1}{2}-\frac{1}{p}}\right. \\
& \leqslant C|\log \epsilon|^{\frac{1}{2}} \epsilon^{\alpha\left(1-\frac{2}{p}\right)},
\end{aligned}
$$

which yields (III.1) with $p=4$.

Step 2. Proof of (III.2). - Recall that, by Proposition II.5, we have

$$
G_{\epsilon}(u, A) \leqslant C|\log \epsilon|
$$

which yields

$$
\frac{1}{2} \int_{B\left(x_{0}, \epsilon^{\alpha / 2}\right) \cap \Omega}\left|\nabla_{A} u\right|^{2}+|d A|^{2}+\frac{1}{2 \epsilon^{2}}\left(1-|u|^{2}\right)^{2} \leqslant C|\log \epsilon| .
$$

By Fubini's theorem, we may hence write

$$
\int_{\epsilon^{\alpha}}^{\epsilon^{\alpha / 2}} I_{r}(u, A) d r \leqslant C|\log \epsilon|
$$

Vol. $12, \mathrm{n}^{\circ} 3-1995$. 
where

$$
I_{r}(u, A)=\frac{1}{2} \int_{\partial B\left(x_{0}, r\right) \cap \Omega}\left|\nabla_{A} u\right|^{2}+|d A|^{2}+\frac{1}{2 \epsilon^{2}}\left(1-|u|^{2}\right)^{2} .
$$

We claim that there is some $\rho \in\left(\epsilon^{\alpha}, \epsilon^{\alpha / 2}\right)$ and a constant $C_{\alpha}$ depending on $\alpha, g$ and $d$ such that

$$
I_{\rho}(u, A) \leqslant \frac{C_{\alpha}}{\rho} .
$$

We argue as in [2] and assume by contradiction that (III.6) does not hold for any $\rho \in\left(\epsilon^{\alpha}, \epsilon^{\alpha / 2}\right)$ and any $C_{\alpha}$. Integrating we obtain

$$
\begin{aligned}
\int_{\epsilon^{\alpha}}^{\epsilon^{\alpha / 2}} I_{r}(u, A) d r & \geqslant \int_{\epsilon^{\alpha}}^{\epsilon^{\alpha / 2}} \frac{C_{\alpha}}{r} d r \\
& \geqslant \frac{1}{2} \alpha C_{\alpha}|\log \epsilon| .
\end{aligned}
$$

This contradicts (III.6) for $C_{\alpha}$ choosen sufficiently large. We distinguish next two cases.

Case 1. $-\partial B\left(x_{0}, \rho\right) \cap \Omega=\emptyset$. We apply proposition II.4 on the ball $B\left(x_{0}, \rho\right)$. This yields by (III.7)

$$
\int_{B\left(x_{0}, \rho\right)} \frac{1}{\epsilon^{2}}\left(1-|u|^{2}\right)^{2}-|h|^{2} \leqslant C \rho I_{\rho}(u, A) \leqslant C .
$$

Combined with (III.1), this yields (III.2) in the case considered.

Case 2. $-\partial B\left(x_{0}, \rho\right) \cap \Omega \neq \emptyset$. We write

$$
\partial\left[B\left(x_{0}, \rho\right) \cap \Omega\right]=\left(\partial B\left(x_{0}, \rho\right) \cap \Omega\right) \cup \Gamma_{\rho}
$$

where

$$
\Gamma_{\rho}=\partial \Omega \cap B\left(x_{0}, \rho\right) .
$$

Let $x_{1}$ be a point in $\Omega$, take $x_{1}$ as the origin and apply proposition II.4 on $\Omega \cap B\left(x_{0}, \rho\right)$. This yield the estimate

$$
\begin{aligned}
& \int_{B\left(x_{0}, \rho\right) \cap \Omega} \frac{1}{\epsilon^{2}}\left(1-|u|^{2}\right)^{2}-|h|^{2} \leqslant C\left(\rho+\left|x_{1}-x_{0}\right|\right) I_{\rho}(u, A) \\
& \quad+\int_{\Gamma_{\rho}}\left(x-x_{1}\right) \cdot \nu\left[\left(\tau \cdot \nabla_{A} u\right)^{2}-\left(\nu \cdot \nabla_{A} u\right)^{2}\right] \\
& \quad-\frac{1}{2} \int_{\Gamma_{\rho}}\left(x-x_{1}\right) \cdot \nu h^{2} \\
& \quad+\int_{\Gamma_{\rho}}\left(x-x_{1}\right) \cdot \tau\left(\tau \cdot \nabla_{A} u, \nu \cdot \nabla_{A} u\right) .
\end{aligned}
$$


(We have used the fact that $|u|=1$ on $\Gamma_{\rho} \subset \partial \Omega$.) We have

$$
\int_{\Gamma_{\rho}}\left(x-x_{1}\right) \cdot \tau\left(\tau \cdot \nabla_{A} u, \nu \cdot \nabla_{A} u\right) \leqslant \frac{1}{2} \int_{\Gamma_{\rho}}\left|\left(x_{1}-x_{0}\right) \cdot \tau\right|\left[\left|\tau . \nabla_{A} u\right|^{2}-\left|\nu \cdot \nabla_{A} u\right|^{2}\right]
$$

Assume that $\epsilon$ is sufficiently small, let $\pi x_{0}$ be the nearest point projection of $x_{0}$ on $\partial \Omega$ and choose $x_{1}$ such that $\operatorname{dist}\left(x_{1}, \pi x_{0}\right)=4 \rho$ and $x_{0} \in\left[\pi x_{0}, x_{1}\right]$. Then we have

$$
\left(x-x_{1}\right) . \nu \geqslant 0 \quad \forall x \in \Gamma_{\rho}
$$

and

$$
\frac{1}{2}\left(x-x_{1}\right) \cdot \tau \leqslant\left(x-x_{1}\right) \cdot \nu \quad \forall x \in \Gamma_{\rho} .
$$

Then (III.8) yields

$$
\begin{aligned}
\int_{B\left(x_{0}, \rho\right) \cap \Omega} \frac{1}{\epsilon^{2}}\left(1-|u|^{2}\right)^{2}-|h|^{2} & \leqslant C \rho\left[I_{\rho}(u, A)+\int_{\Gamma_{\rho}}\left|\tau . \nabla_{A} u\right|^{2}\right] \\
& \leqslant C \rho\left[I_{\rho}(u, A)+\int_{\Gamma_{\rho}} g^{2}\right] \leqslant C,
\end{aligned}
$$

and this implies (III.2).

\section{LOCATING THE SINGULARITIES}

The purpose of this section is to extend lemma IV.1 of [2] to our situation. More precisely we are going to give a first description of the vortices and their location. We will prove

THEOREM IV.1. - There exist constants $N \in \mathbb{N}, \lambda_{0}>0$ depending only on $g, d, \Omega$ (and not on $\epsilon$ ), and points $x_{1}^{\epsilon}, \ldots, x_{i}^{\epsilon}, \ldots, x_{N_{\epsilon}}^{\epsilon}$ in $\Omega$ such that

$$
N_{\epsilon} \leqslant N
$$

and

$$
\left|u_{\epsilon}(x)\right| \geqslant \frac{1}{2} \quad \text { for } \quad x \in \Omega \backslash\left(\bigcup_{i=1}^{N_{\epsilon}} B\left(x_{i}^{\epsilon}, \lambda_{0} \epsilon\right)\right) .
$$

In this section also, if not otherwise necessary, we drop the indice $\epsilon$ for $u_{\epsilon}, h_{\epsilon}, A_{\epsilon}$ and $x_{i}^{\epsilon}$ and simply denote $u, h, A$ and $x_{i}$. We assume throughout that $\epsilon<1$.

Vol. 12, $\mathrm{n}^{\circ}$ 3-1995. 
Our analysis here, follows closely the ideas of [2]. The main difference in our situation is that we only have the local estimate (III.2) (compare with Theorem III.3 of [2]).

The next result, as in [2], plays a crucial role:

PROPOSITION IV.1. - There exists positive constants $\lambda_{0}, \mu_{0}$ depending only on $d, g$ and $\Omega$ such that if $\left(u_{\epsilon}, A_{\epsilon}\right)$ is a minimizer of $G_{\epsilon}$ on $V$ satisfying

$$
\int_{B\left(x_{0}, 2 l\right) \cap \Omega} \frac{1}{\epsilon^{2}}\left(1-\left|u_{\epsilon}\right|^{2}\right)^{2} \leqslant \mu_{0}
$$

with $x_{0} \in \Omega$ and

$$
\frac{l}{\epsilon} \geqslant \lambda_{0}, \quad l \leqslant 1,
$$

then

$$
\left|u_{\epsilon}(x)\right| \geqslant \frac{1}{2} \quad \text { on } \quad \Omega \cap B\left(x_{0}, l\right)
$$

Proof. - The argument is exactly the same as the proof of the theorem III.4 of [2]. Indeed, observe that

$$
|\nabla| u|| \leqslant\left|\nabla_{A} u\right|
$$

[If $|u| \neq 0$, we may write locally $u=|u| e^{i \phi}$, and we compute

$$
\nabla_{A} u=(\nabla|u|+i|u|(\nabla \phi-A)) e^{i \phi}
$$

and (IV.6) follows. Otherwise if $|u|=0$

$$
\nabla_{A} u=\nabla u
$$

and (IV.6) follows similarly]. By proposition II.6 we have therefore

$$
|\nabla| u|| \leqslant C / \epsilon
$$

and use the argument of [2].

Next we will (using proposition III.1) first give a local version of theorem IV.1.

Proposition IV.2. - Let $0<\alpha<1$, and $x_{0} \in \Omega$ be given. There exists a constant $\bar{N}_{\alpha}$ depending only on $\alpha, g$, and $d$, points $x_{1}^{\epsilon}, \ldots, x_{l_{\epsilon}}^{\epsilon}$ in $B\left(x_{0}, \epsilon^{\alpha}\right) \cap \Omega$ such that

$$
l_{\epsilon} \leqslant \bar{N}_{\alpha}
$$


and

$$
\left|u_{\epsilon}(x)\right| \geqslant \frac{1}{2} \quad \text { for } \quad x \in B\left(x_{0}, \epsilon^{\alpha}\right) \cap\left(\Omega \backslash\left(\bigcup_{i=1}^{l_{\epsilon}} B\left(x_{i}^{\epsilon}, \lambda_{0} \epsilon\right)\right)\right)
$$

Proof. - We follow the proof of lemma IV.4 of [2]. Consider a family of $\operatorname{discs}\left(B\left(x_{i}, \lambda_{0} \epsilon\right)\right)_{i \in I}$, where $\lambda_{0}$ is the constant in proposition IV.1 such that

$$
\begin{aligned}
& x_{i} \in \Omega \cap B\left(x_{0}, \epsilon^{\alpha}\right) \\
& B\left(x_{i}, \frac{\lambda_{0} \epsilon}{4}\right) \cap B\left(x_{j}, \frac{\lambda_{0} \epsilon}{4}\right)=\emptyset \quad \forall i \neq j \\
& \Omega \cap B\left(x_{0}, \epsilon^{\alpha}\right) \subset \bigcup_{i \in I} B\left(x_{i}, \lambda_{0} \epsilon\right) .
\end{aligned}
$$

We say that $B\left(x_{i}, \lambda_{0} \epsilon\right)$ is a bad disc if

$$
\int_{B\left(x_{i}, 2 \lambda_{0} \epsilon\right) \cap \Omega} \frac{1}{\epsilon^{2}}\left(1-\left|u_{\epsilon}\right|^{2}\right)^{2} \geqslant \mu_{0} .
$$

Hence if $x$ does not belong to a bad disc, we deduce, from proposition IV.1 that

$$
|u(x)| \geqslant 1 / 2
$$

There is an absolute constant $C$ such that

$$
\sum_{i \in I} \int_{B\left(x_{i}, 2 \lambda_{0} \epsilon\right) \cap \Omega}\left(1-\left|u_{\epsilon}\right|^{2}\right)^{2} \leqslant C \int_{B\left(x_{0}, \epsilon^{\alpha}\right) \cap \Omega}\left(1-\left|u_{\epsilon}\right|^{2}\right)^{2} .
$$

Therefore the number of bad discs is bounded by

$$
\frac{C}{\epsilon^{2}} \int_{B\left(x_{0}, \epsilon^{\alpha}\right) \cap \Omega}\left(1-\left|u_{\epsilon}\right|^{2}\right)^{2}
$$

and the conclusion follows from proposition III.1.

The last ingredient in the proof of theorem IV.1 is to show that the contribution of a bad disc to the total energy is of the order of $|\log \epsilon|$. Hence in view of proposition II.5 there cannot be many bad discs.

Proposition IV.3. - Let $0<\alpha<1$ and $x_{0} \in \Omega$ be given. Assume that

$$
\left|u_{\epsilon}\left(x_{0}\right)\right|<\frac{1}{2}
$$


There exists a constant $C_{\alpha}$ depending only on $\alpha, g$, and $d$ such that

$$
\int_{B\left(x_{0}, \epsilon^{\alpha}\right) \cap \Omega}\left|\nabla_{A_{\epsilon}} u_{\epsilon}\right|^{2} \geqslant C_{\alpha}|\log \epsilon|
$$

Proof. - In order to prove proposition IV.3 we need the following lemma.

LEMMA IV.1. - Let $D>0,0<\beta<1, \gamma>1$ be given constants, such that $\gamma \beta<1$. Let $\rho<\epsilon^{\beta}$. Assume that $\rho^{\gamma}>\lambda_{0} \epsilon$, that

$$
\begin{gathered}
I_{\rho}\left(u_{\epsilon}, A_{\epsilon}\right)<\frac{D}{\rho}, \\
\left|u_{\epsilon}\right| \geqslant \frac{1}{2} \quad \text { on } \quad \partial B\left(x_{0}, \rho\right) \cap \Omega,
\end{gathered}
$$

and

$$
\operatorname{deg}\left(\frac{u_{\epsilon}}{\left|u_{\epsilon}\right|}, \partial\left[B\left(x_{0}, \rho\right) \cap \Omega\right]\right)=0 .
$$

Then we have

$$
\left|u_{\epsilon}\right| \geqslant \frac{1}{2} \quad \text { on } \quad B\left(x_{0}, \rho^{\gamma}\right) \cap \Omega,
$$

provided $\epsilon<\epsilon_{0}$, for some constant $\epsilon_{0}$ depending on $D, \beta, \gamma, g$, $d$ and $\Omega$.

We postpone the proof of lemma IV.1 and complete the proof of proposition IV.3.

Proof of proposition IV.3 completed. - Set

$$
\delta_{\alpha}=\frac{\alpha^{\frac{1}{2}}-\alpha}{2\left(\bar{N}_{\alpha}+1\right)}
$$

for $k=1, \ldots, 2\left(\bar{N}_{\alpha}+1\right)$, consider the intervals

$$
I_{k}=\left(\epsilon^{\alpha^{1 / 2}-k \delta_{\alpha}}, \epsilon^{\alpha^{1 / 2}-(k+1) \delta_{\alpha}}\right),
$$

and set

$$
\mathcal{C}_{k}=\left[B\left(x_{0}, \epsilon^{\alpha^{1 / 2}-k \delta_{\alpha}}\right) \backslash B\left(x_{0}, \epsilon^{\alpha^{1 / 2}-(k+1) \delta_{\alpha}}\right)\right] \cap \Omega .
$$

We apply proposition IV.2 to the domain $B\left(x_{0}, \epsilon^{\alpha}\right) \cap \Omega$. Let $x_{1}, \ldots, x_{i}, \ldots, x_{l_{c}}$ be as in proposition IV.2. We claim that, if $\epsilon<\epsilon_{0}$ (depending on $\alpha, g, d$, $\Omega)$ then there is some $k_{0}$ in $\left\{1, \ldots, 2\left(\bar{N}_{\alpha}+1\right)\right\}$ such that

$$
\mathcal{C}_{k_{0}} \cap\left(\bigcup_{i=1}^{l_{\epsilon}} B\left(x_{i}, \lambda_{0} \epsilon\right)\right)=\emptyset \text {. }
$$


The proof of (IV.18) relies on a simple counting argument. Indeed, since $l_{\epsilon} \leqslant \bar{N}_{\alpha}$, the union of intervals

$$
\bigcup_{i=1 \ldots l_{\varepsilon}}\left(\left|x_{i}-x_{0}\right|-\lambda_{0} \epsilon,\left|x_{i}-x_{0}\right|+\lambda_{0} \epsilon\right)
$$

cannot intersect all the $I_{k}$. It follows in particular from (IV.18) that

$$
|u(x)| \geqslant \frac{1}{2} \quad \forall x \in \mathcal{C}_{k_{0}} .
$$

Therefore, for any $\rho \in I_{k_{0}}$

$$
d_{k_{0}}=\operatorname{deg}\left(\frac{u_{\epsilon}}{\left|u_{\epsilon}\right|}, \partial\left[B\left(x_{0}, \rho\right) \cap \Omega\right]\right)
$$

is well defined and does not depend on $\rho$. We complete the proof in two steps.

Step 1. - We have

$$
d_{k_{0}} \neq 0
$$

Indeed assume by contradiction that $d_{k_{0}}=0$. Adapting the argument of the proof of proposition III.1, we would find some $\rho_{0}$ in $I_{k_{0}}$ such that

$$
I_{\rho_{0}}(u, A)<\frac{C}{\rho_{0}}
$$

where $C$ depends only on $\alpha, g, d$, and $\Omega$. Applying lemma IV.1 to $B\left(x_{0}, \rho_{0}\right) \cap \Omega$ we are led to $\left|u\left(x_{0}\right)\right| \geqslant \frac{1}{2}$, a contradiction.

Step 2. - Proof of (IV.13). Choose $(\tilde{u}, \tilde{A})$, gauge equivalent to $(u, A)$ satifying

$$
\left\{\begin{array}{lll}
d^{*} \tilde{A}=0 & \text { in } & B\left(x_{0}, \epsilon^{\alpha}\right) \cap \Omega \\
\tilde{A} . \nu=0 & \text { on } & \partial B\left(x_{0}, \epsilon^{\alpha}\right) \cap \Omega \\
\tilde{A} . \tau=0 & \text { on } & B\left(x_{0}, \epsilon^{\alpha}\right) \cap \partial \Omega .
\end{array}\right.
$$

We may find $\tilde{A}$ by introducing the solution $\tilde{\xi}$ of

$$
\left\{\begin{array}{l}
\Delta \tilde{\xi}=h \quad \text { in } \quad B\left(x_{0}, \epsilon^{\alpha}\right) \cap \Omega \\
\tilde{\xi}=0 \quad \text { on } \quad \partial B\left(x_{0}, \epsilon^{\alpha}\right) \cap \Omega \\
\frac{\partial \tilde{\xi}}{\partial \nu}=0 \quad \text { on } \quad B\left(x_{0}, \epsilon^{\alpha}\right) \cap \partial \Omega
\end{array}\right.
$$

Vol. $12, \mathrm{n}^{\circ} 3-1995$. 
so that $\tilde{A}=\left(-\tilde{\xi}_{x_{2}}, \tilde{\xi}_{x_{1}}\right)$. Note that the domain $B\left(x_{0}, \epsilon^{\alpha}\right) \cap \Omega$ may have corners. However by a result of Grisvard [10], we may nevertheless assert that

$$
\|\nabla \xi\|_{L^{2}}+\left\|\nabla^{2} \xi\right\|_{L^{2}\left(B\left(x_{0}, \epsilon^{\alpha}\right) \cap \Omega\right)} \leqslant C\|h\|_{L^{2}\left(B\left(x_{0}, \epsilon^{\alpha}\right) \cap \Omega\right)},
$$

and hence, we deduce that

$$
\int_{B\left(x_{0}, \epsilon^{\alpha}\right) \cap \Omega}|\tilde{A}|^{2}+|\nabla \tilde{A}|^{2} \leqslant \int_{B\left(x_{0}, \epsilon^{\alpha}\right) \cap \Omega}|h|^{2} \leqslant C .
$$

We have

$$
\forall \rho \in I_{k_{0}} \quad\left|d_{k_{0}}\right|=\frac{1}{2 \pi}\left|\int_{\partial\left[B\left(x_{0}, \rho\right) \cap \Omega\right]} \frac{1}{|u|^{2}}\left(\tilde{u} \times \frac{\partial \tilde{u}}{\partial \tau}\right)\right| \geqslant 1 .
$$

On $B\left(x_{0}, \epsilon^{\alpha}\right) \cap \partial \Omega$, we have $\tilde{A} \cdot \tau=0$. Hence

$$
\frac{\partial \tilde{u}}{\partial \tau}=i g \tilde{u} \quad \text { on } \quad B\left(x_{0}, \epsilon^{\alpha}\right) \cap \partial \Omega
$$

and

$$
\left|\int_{B\left(x_{0}, \rho\right) \cap \partial \Omega}\left(\tilde{u} \times \frac{\partial \tilde{u}}{\partial \tau}\right)\right|=\left|\int_{B\left(x_{0}, \rho\right) \cap \partial \Omega} g\right| \leqslant C \epsilon^{\alpha} .
$$

Combining (IV.24) and (IV.25) we are led to

$$
\left|\int_{\partial B\left(x_{0}, \rho\right) \cap \Omega} \frac{1}{|u|^{2}}\left(\tilde{u} \times \frac{\partial \tilde{u}}{\partial \tau}\right)\right| \geqslant 2 \pi\left(1-C \epsilon^{\alpha}\right) \geqslant \pi .
$$

By Cauchy-Schwarz inequality, we deduce that

$$
\forall \rho \in I_{k_{0}} \quad \int_{\partial B\left(x_{0}, \rho\right) \cap \Omega}|\nabla \tilde{u}|^{2} \geqslant \frac{C}{\rho},
$$

and hence integrating on $I_{k_{0}}$ we are led to

$$
\int_{\mathcal{C}_{k_{0}}}|\nabla \tilde{u}|^{2} \geqslant C|\log \epsilon|
$$

Combining this with (IV.23) we deduce that

$$
\int_{\mathcal{C}_{k_{0}}}\left|\nabla_{\tilde{A}} \tilde{u}\right|^{2}=\int_{\mathcal{C}_{k_{0}}}\left|\nabla_{A} u\right|^{2} \geqslant C_{\alpha}|\log \epsilon|,
$$

and this yields the conclusion. 
We now turn to the proof of lemma IV.1.

Proof of lemma IV.1. - It is convenient to introduce the following notation. For any subset $K$ of $\Omega$, set

$$
G(u, A, K)=\frac{1}{2} \int_{K}\left|\nabla_{A} u\right|^{2}+|d A|^{2}+\frac{1}{2 \epsilon^{2}}\left(1-|u|^{2}\right)^{2} .
$$

We claim that

$$
G\left(u, A, \Omega \cap B\left(x_{0}, \rho\right)\right) \leqslant C\left(\rho\left[I_{\rho}(u, A)\right]+o(1)\right)
$$

where $C$ is a constant depending only on $g, d, \Omega$, and $\beta$ and $o(1) \rightarrow 0$ uniformly as $\epsilon \rightarrow 0$.

Proof of (IV.27). - The idea is to construct a comparison function, and we adopt the construction of [1]. To that aim we are going to change the gauge, and consider $(\tilde{u}, \tilde{A})$ gauge equivalent to $(u, A)$ such that

$$
\left\{\begin{array}{l}
d^{*} \tilde{A}=0 \\
\tilde{A} \cdot \tau=\frac{1}{|\Gamma|} \int_{U}^{\text {on }} h \quad \text { on } \quad \Gamma .
\end{array}\right.
$$

Here we have used the notation

$$
U=B\left(x_{0}, \epsilon^{\beta}\right) \cap \Omega
$$

and

$$
\Gamma=\partial U=\partial\left[B\left(x_{0}, \epsilon^{\beta}\right) \cap \Omega\right] .
$$

This gauge $\tilde{A}$ can be found by introducing the solution $\tilde{\xi}$ to

$$
\left\{\begin{array}{l}
\Delta \tilde{\xi}=h \quad \text { on } \quad U \\
\frac{\partial \tilde{\xi}}{\partial \nu}=\frac{1}{|\Gamma|} \int_{U} h \quad \text { on } \quad \Gamma \\
\int_{U} \tilde{\xi}=0
\end{array}\right.
$$

so that $\tilde{A}=\left(-\tilde{\xi}_{x_{2}}, \tilde{\xi}_{x_{1}}\right)$ in $U, \tilde{A}$ is extended to $\Omega$ as in the proof of proposition I.2.

We easily verify that

$$
\int_{U}|\nabla \tilde{\xi}|^{2} \leqslant C \int_{U}|h|^{2}
$$


and hence by proposition III.1

$$
\int_{U}|\tilde{A}|^{2} \leqslant C \epsilon^{\beta}|\log \epsilon| \longrightarrow 0
$$

Since $\int_{\Gamma} \tilde{A} . \tau=\int_{U} h \rightarrow 0$ as $\epsilon \rightarrow 0$, we deduce, from assumption (IV.14) that

$$
\int_{\Gamma}\left|\frac{\partial \tilde{u}}{\partial \tau}\right|^{2} \leqslant 2 \int_{\Gamma}\left|\nabla_{A} u\right|^{2}+\int_{\Gamma}|\tilde{A} \cdot \tau|^{2} .
$$

We have by (IV.28)

$$
\begin{aligned}
\int_{\Gamma}|\tilde{A} \cdot \tau|^{2} \leqslant \frac{1}{|\Gamma|}\left(\int_{U}|h|\right)^{2} & \leqslant \frac{1}{|\Gamma|} \int_{\Omega}|h|^{2} \operatorname{mes}|U| \\
& \leqslant C \epsilon^{\beta} \int_{\Omega}|h|^{2} \leqslant C \epsilon^{\beta}|\log \epsilon| .
\end{aligned}
$$

Hence

$$
\int_{\Gamma}\left|\frac{\partial \tilde{u}}{\partial \tau}\right|^{2} \leqslant \frac{C}{\rho}+o(1)
$$

where $C$ is a constant depending on $D$. Since the degree of $\tilde{u}$ restricted to $\Gamma$ is zero, we may write on $\Gamma$

$$
\tilde{u}=|u| e^{i \tilde{\phi}}
$$

where $\tilde{\phi}$ is a smooth map from $\Gamma$ to $\mathbb{R}$.

We are now able to introduce a comparison function $(v, B)$. We first set

$$
B=\tilde{A} \quad \text { in } \Omega
$$

and

$$
\left\{\begin{array}{l}
v=\eta e^{i \psi} \quad \text { in } \quad U \\
v=\tilde{u} \quad \text { in } \quad \Omega \backslash U
\end{array}\right.
$$

Here $\psi$ represents the solution of

$$
\left\{\begin{array}{l}
\Delta \psi=0 \quad \text { in } \quad U \\
\psi=\tilde{\phi} \quad \text { on } \quad \Gamma=\partial U
\end{array}\right.
$$

and $\eta$ the solution of

$$
\left\{\begin{array}{l}
-\epsilon^{2} \Delta \eta+\eta=1 \quad \text { in } \quad U \\
\eta=|u| \quad \text { on } \quad \Gamma=\partial U
\end{array}\right.
$$


VORTICES FOR A VARIATIONAL PROBLEM RELATED TO SUPERCONDUCTIVITY 273

Arguing as in [1], (Theorem 2) we may assert by (IV.31) that

$$
\begin{gathered}
\int_{U}|\nabla \psi|^{2} \leqslant C\left[\rho I_{\rho}(u, A)\right]+o(1), \\
\int_{U}|\nabla \eta|^{2} \leqslant C \epsilon^{1 / 2}\left[\rho I_{\rho}(u, A)\right]+o(1), \\
\frac{1}{\epsilon^{2}} \int_{U}(1-\eta)^{2} \leqslant C \epsilon^{1 / 2}\left[\rho I_{\rho}(u, A)\right]+o(1),
\end{gathered}
$$

and hence

$$
\frac{1}{2} \int_{U}|\nabla v|^{2}+\frac{1}{4 \epsilon^{2}} \int_{U}\left(1-|v|^{2}\right)^{2} \leqslant C\left[\rho I_{\rho}(u, A)\right]+o(1) \leqslant C .
$$

Combining this with (IV.30) and proposition III.1 we deduce, from the definition of $(v, B)$ that

$$
G(u, A, U) \leqslant G(v, B, U) \leqslant C
$$

This complete the proof of (IV.27).

We deduce from (IV.27) that

$$
\int_{\rho^{\gamma}}^{\rho} I_{r}(u, A) d r \leqslant C
$$

and hence, arguing as in the proof of proposition III.2, we deduce that there is some $r_{0} \in\left[\rho^{\gamma}, \rho\right]$ such that

$$
I_{r_{0}}(u, A) \leqslant \frac{C}{r_{0}\left|\log r_{0}\right|^{\frac{1}{2}}}
$$

where $C$ does not depend on $\epsilon$. Arguing as in the proof of (IV.27) we might now assert that

$$
\begin{aligned}
G\left(u, A, B\left(x_{0}, r_{0}\right) \cap \Omega\right) & \leqslant C\left(\left[r_{0} I_{r_{0}}(u, A)\right]+o(1)\right) \\
& \leqslant \frac{C}{\left|\log r_{0}\right|^{\frac{1}{2}}}+o(1) \leqslant \frac{C}{|\log \epsilon|^{\frac{1}{2}}}+o(1) .
\end{aligned}
$$

In particular

$$
\frac{1}{\epsilon^{2}} \int_{\Omega \cap B\left(x_{0}, \rho^{\gamma}\right)}\left(1-|u|^{2}\right)^{2} \leqslant \frac{C}{|\log \epsilon|^{\frac{1}{2}}}+o(1) \quad \longrightarrow 0, \quad \text { as } \epsilon \rightarrow 0
$$

and the conclusion of Lemma IV.1 follows from proposition IV.1. 
Proof of theorem IV.1. - Let $\left(x_{i}\right)_{i \in I}$ be a finite family of points in $\Omega$, as in the proof of proposition IV.1, satisfying

$$
\begin{aligned}
& x_{i} \in \Omega \\
& B\left(x_{i}, \frac{\lambda_{0} \epsilon}{4}\right) \bigcap B\left(x_{j}, \frac{\lambda_{0} \epsilon}{4}\right)=\emptyset \quad \forall i \neq j \\
& \Omega \subset \bigcup_{i \in I} B\left(x_{i}, \lambda_{0} \epsilon\right) .
\end{aligned}
$$

We say that $B\left(x_{i}, \lambda_{0} \epsilon\right)$ is a bad disc (in this proof) if there is some $y_{i} \in B\left(x_{i}, \lambda_{0} \epsilon\right)$ such that

$$
\left|u\left(y_{i}\right)\right|<\frac{1}{2}
$$

We denote by $J$ the set of indices for bad discs. In order to prove theorem IV.1, it suffices to show that

$$
\# J<C,
$$

where $C$ is some constant depending only on $g, d$, and $\Omega$. To that aim, let $0<\alpha<1$ be fixed, and consider the balls $B\left(y_{i}, \epsilon^{\alpha}\right)$, for $i \in J$ (where $y_{i}$ is one of the points verifying (IV.35) in the bad ball $B\left(x_{i}, \lambda_{0} \epsilon\right)$ ). Clearly, by (IV.35) and proposition IV.3, we have

$$
\int_{B\left(y_{i}, \epsilon^{\alpha}\right) \cap \Omega}\left|\nabla_{A} u\right|^{2} \geqslant C_{\alpha}|\log \epsilon| .
$$

Set

$$
W=\bigcup_{i \in J} B\left(x_{i}, 2 \epsilon^{\alpha}\right) \cap \Omega .
$$

We claim that

$$
\begin{aligned}
\int_{W}\left|\nabla_{A} u\right|^{2} & \geqslant C \sum_{i \in J} \int_{B\left(x_{i}, 2 \epsilon^{\alpha}\right) \cap \Omega}\left|\nabla_{A} u\right|^{2} \\
& \geqslant C(\# J)|\log \epsilon|
\end{aligned}
$$

where $C$ is a constant depending only on $\alpha, g, d$ and $\Omega$.

Proof of (IV.38). - It suffices to bound (independently of $\epsilon$ ) the number of balls $B\left(x_{i}, 2 \epsilon^{\alpha}\right)$ to which any point $y$ in $W$ may belong. Let $y \in W$ and consider the subset $K_{y}$ of indices of $J$ such that

$$
K_{y}=\left\{i \in J, \quad y \in B\left(y_{i}, 2 \epsilon^{\alpha}\right)\right\} .
$$


It follows that, for $i$ in $K_{y}$ we have (provided $\epsilon$ is sufficiently small)

$$
x_{i} \in B\left(y, 2 \epsilon^{\alpha}\right) .
$$

On the other hand, we deduce, from proposition IV.2, that the number of such $x_{i}$ is bounded by a constant depending just on $\alpha, g, d$ and $\Omega$, hence

$$
\# K_{y}<C,
$$

and this yields (IV.38). Combining (IV.38) with proposition II.5 we deduce (IV.36) and complete the proof of the theorem.

\section{GLOBAL ESTIMATES}

At this point we had only a local estimate for the magnetic field. Thanks to theorem IV.1, we will be able to derive an equation for $h_{\epsilon}$ which leads directly to global estimates. We are going to prove in this section.

THEOREM V.1. - Given $1<p<2$ and $q \geqslant 1$ there are constants $C_{p}$ (resp. $C_{q}$ ) depending only on $d, g, \Omega$ and $p$ (resp. q) such that

$$
\begin{array}{lc}
\int_{\Omega}\left|\nabla h_{\epsilon}\right|^{p} \leqslant C_{p} & \forall p<2, \\
\int_{\Omega}\left|h_{\epsilon}\right|^{q} \leqslant C_{q} & \forall q<+\infty, \\
\int_{\Omega}\left|\nabla_{A_{\epsilon}} u_{\epsilon}\right|^{p} \leqslant C_{p} & \forall p<2 .
\end{array}
$$

\subsection{The equation for $h$}

Recall that we have found (Theorem IV.1) $N_{\epsilon}$ points $x_{1}^{\epsilon}, \ldots, x_{N_{\epsilon}}^{\epsilon}$ in $\Omega$ such that

$$
N_{\epsilon} \leqslant N
$$

and

$$
|u(x)| \geqslant \frac{1}{2} \quad \text { on } \quad \Omega \backslash \bigcup_{i=1 \ldots N_{\epsilon}} B\left(x_{i}^{\epsilon}, \lambda_{0} \epsilon\right) .
$$

Of course the limits as $\epsilon \rightarrow 0$ of the points $x_{i}^{\epsilon}$ are the good candidates for being the vortices of $u_{*}$. In theorem IV.1 we have not excluded the Vol. $12, n^{\circ} 3-1995$. 
possibility that some of the discs $B\left(x_{i}^{\epsilon}, \lambda_{0} \epsilon\right)$ may intersect. To avoid this unpleasant situation we will argue as in [2] (lemma X.3) and replace the discs $B\left(x_{i}^{\epsilon}, \lambda_{0} \epsilon\right)$ by slightly larger discs $B\left(x_{i}^{\epsilon}, \lambda \epsilon\right)$ for $i \in J^{\epsilon}$ a subset of $\left\{1, \ldots, l_{\epsilon}\right\}$ and where $\lambda>\lambda_{0}$ is another constant depending on $g, \Omega$ and such that

$$
\begin{gathered}
B\left(x_{i}^{\epsilon}, \lambda \epsilon\right) \cap B\left(x_{i}^{\epsilon}, \lambda \epsilon\right)=\emptyset \quad \forall i \neq j, \\
|u(x)| \geqslant \frac{1}{2} \quad \text { if } \quad \bigcup_{i \in J^{\epsilon}} B\left(x_{i}^{\epsilon}, \lambda \epsilon\right) .
\end{gathered}
$$

In this section also we drop the indice $\epsilon$ when no confusion is possible.

We set

$$
\omega_{i}=B\left(x_{i}, \lambda \epsilon\right) \quad \text { for } \quad i \in J
$$

and

$$
\Gamma_{i}=\partial B\left(x_{i}, \lambda \epsilon\right) \quad \text { for } \quad i \notin J .
$$

We consider the subset $J_{1}$ of $J$ defined by

$$
J_{1}=\left\{i \in J, B\left(x_{i}, \lambda \epsilon\right) \cap \partial \Omega=\emptyset\right\}
$$

and

$$
J_{2}=J \backslash J_{1}
$$

Set

$$
\begin{aligned}
& \tilde{\Omega}=\Omega \backslash \bigcup_{i \in J} B\left(x_{i}, \lambda \epsilon\right), \\
& \hat{\Omega}=\Omega \backslash \bigcup_{i \in J} B\left(x_{i}, 2 \lambda \epsilon\right), \\
& \Omega_{1}=\Omega \backslash \bigcup_{i \in J_{2}} B\left(x_{i}, \lambda \epsilon\right),
\end{aligned}
$$

and

$$
\Gamma_{0}=\partial \Omega_{1} .
$$

Proposition V.1. - We have on $\tilde{\Omega}$

$$
\star d\left(i \frac{u_{\epsilon}}{\left|u_{\epsilon}\right|^{2}}, d_{A_{\epsilon}} u_{\epsilon}\right)=-h_{\epsilon} .
$$

(Recall that $d_{A} u=d u-i A u$, where $A$ is considered as a form.) 
Proof. - Since $|u| \geqslant 1 / 2$ on $\tilde{\Omega}$ and since (V.6) is a local property it suffices to verify it locally. We may write

$$
u=|u| e^{i \phi} \quad \text { locally on } \quad \tilde{\Omega}
$$

and hence

$$
\left(i u, d_{A} u\right)=|u|^{2}(d \phi-A) \text {. }
$$

Thus

$$
d\left(i \frac{u}{|u|^{2}}, d_{A} u\right)=-d A=-\star h .
$$

Proposition V.2. - Set $\rho_{\epsilon}=\left|u_{\epsilon}\right|$. The magnetic field $h_{\epsilon}$ satisfies

$$
-\operatorname{div}\left(\frac{1}{\rho_{\epsilon}^{2}} \nabla h_{\epsilon}\right)+h_{\epsilon}=0 \quad \text { in } \quad \tilde{\Omega} .
$$

Proof. - Recall the Ginzburg-Landau equation

$$
-\star d h=\left(i u, d_{A} u\right)
$$

Hence in $\tilde{\Omega}$ we may write

$$
\star d\left(\star \frac{1}{\rho^{2}} d h\right)=-\star d\left(i \frac{u}{|u|^{2}}, d_{A} u\right)=h .
$$

Equation (V.7) is supplemented with the boundary condition

$$
\frac{\partial h}{\partial \nu}=-g \quad \text { on } \quad \partial \Omega
$$

This leads us to consider elliptic equations of divergence form with Neumann boundary condition. As in [2] we will use the method of Stampacchia [15] to derive $L^{p}$ bounds for $|\nabla h|$ with $p<2$. This is the purpose of the next section.

\subsection{Auxiliary linear problems}

In this section we consider a domain $\Omega \subset \mathbb{R}^{2}$, and a positive function $a: \Omega \rightarrow \mathbb{R}$, and a constant $1>\gamma>0$ such that

$$
\gamma<a<\gamma^{-1}
$$


Let $f=\left(f_{1}, f_{2}\right)$ be a vector field in $L^{q}(\Omega)$, for some $q>2$. We consider the solution $\xi \in H^{1}(\Omega)$ of the linear problem

$$
\int_{\Omega} a \nabla \xi \nabla \phi+\xi \phi=\int_{\Omega} f \nabla \phi \quad, \quad \forall \phi \in H^{1}(\Omega) .
$$

Clearly $\xi$ is unique and can be found by minimization.

Testing with $\phi \equiv 1$, we obtain

$$
\int_{\Omega} \xi=0
$$

and testing with $\phi=\xi$, we derive

$$
\int_{\Omega}|\nabla \xi|^{2}+\xi^{2} \leqslant C \int_{\Omega}|f|^{2}
$$

where $C$ depends on $\alpha$. Our main estimate is the following.

Proposition V.3. - For any $q>2$ we have

$$
\|\xi\|_{L^{\infty}(\Omega)} \leqslant C_{q}\|f\|_{L^{q}(\Omega)}
$$

where $C_{q}$ is a constant depending only on $\alpha, \Omega$, and $q$.

Proof. - The proof is due to G. Stampacchia [15] (in the case of Dirichlet boundary conditions). We closely follow his arguments (as in [2]).

To avoid problems with regularity we assume first that $f$ and $a$ are smooth and derive (V.13) in that case. The general case will then follow by density. Since $f$ and $a$ are smooth, $\xi$ will be also.

For $\delta>0$ consider the set

$$
F(\delta)=\{x \in \Omega, \operatorname{dist}(x, \partial \Omega)<\delta\}
$$

and

$$
\Lambda(\delta)=\partial F(\delta) \backslash \partial \Omega
$$

Let $\pi: F(\delta) \rightarrow \partial \Omega$ be the nearest point projection onto $\partial \Omega$. There is some $\delta_{0}$ such that for $\delta<\delta_{0}, \pi$ is a smooth fibration. Consider the reflexion map

$$
\begin{aligned}
T: F\left(\delta_{0}\right) & \longrightarrow \mathbb{R}^{2} \backslash \Omega \\
x & \longrightarrow T(x),
\end{aligned}
$$

where $T(x)$ is such that $\operatorname{dist}(T(x), \partial \Omega)=\operatorname{dist}(x, \partial \Omega)$ and $x-T(x)$ is parallel to $x-\pi(x)$. Clearly $T$ is smooth and

$$
\|\nabla T\|_{L^{\infty}(F(\delta))} \leqslant C, \quad \forall \delta<\delta_{0} .
$$


Next we consider the level sets of $\xi$. For $k>0$ set

$$
\begin{gathered}
\Omega(k)=\{x \in \Omega, \quad \xi>k\}, \\
\mu(k)=|\Omega(k)|,
\end{gathered}
$$

and

$$
V(k)=\partial \Omega(k) .
$$

We now divide the proof in several steps.

Step 1. - There is some constant $D_{1}$ depending only on $\Omega, \gamma$, and $k_{0} \in\left(D_{1}|f|_{L^{2}}, 2 D_{1}|f|_{L^{2}}\right)$ such that $V\left(k_{0}\right)$ is smooth and

$$
\mathcal{H}^{1}\left(V\left(k_{0}\right)\right) \leqslant \frac{1}{4} \delta_{0}
$$

Where $\mathcal{H}^{1}$ denotes the 1-dimensional Hausdorff measure on $\mathbb{R}^{2}$.

Proof. - By the coarea formula, for any $D_{1}>0$

$$
\begin{aligned}
\int_{D_{1}|f|_{L^{2}}}^{2 D_{1}|f|_{L^{2}}} \mathcal{H}^{1}(V(k)) d k & \leqslant \int_{\Omega}|\nabla \xi| \\
& \leqslant C\left(\int_{\Omega}|\nabla \xi|^{2}\right)^{\frac{1}{2}} \leqslant C|f|_{L^{2}} .
\end{aligned}
$$

We deduce that there is some $k_{0} \in\left(D_{1}|f|_{L^{2}}, 2 D_{1}|f|_{L^{2}}\right)$ such that $V\left(k_{0}\right)$ is smooth (by Sard's theorem, since $\xi$ is smooth) and

$$
\mathcal{H}^{1}\left(V\left(k_{0}\right)\right) \leqslant C / D_{1}
$$

Step 2. - There is a constant $\delta$ depending only on $\Omega$ such that for $k>k_{0}$, the following inequality holds

$$
\gamma \delta\left\|(\xi-k)^{+}\right\|_{L^{2}(\Omega)} \leqslant\left\|\nabla(\xi-k)^{+}\right\|_{L^{1}(\Omega)}
$$

Proof. - We consider the connected components of $V\left(k_{0}\right)$. And the maximal curves $V^{1}\left(k_{0}\right), \ldots, V^{p}\left(k_{0}\right)$. We divide these components in two sets

$$
\begin{gathered}
K_{1}=\left\{i \in\{1, \ldots, p\} V^{i}\left(k_{0}\right) \cap \partial \Omega=\emptyset\right\} \\
K_{2}=\{1, \ldots, p\} \backslash K_{1} .
\end{gathered}
$$

Let $W_{i}$ be the domains bounded by the curves $V^{i}$. Clearly, for $k>k_{0}$, we have

$$
\Omega(k) \subset \Omega\left(k_{0}\right) \subset \cup_{i=1}^{p} W_{i} .
$$

Vol. $12, \mathrm{n}^{\circ} 3-1995$. 
For $i \in K_{1}$ we easily verify that

$$
(\xi-k)^{+}=0 \quad \text { on } \quad \partial W_{i} \quad \text { for } i \in K_{1} \quad k>k_{0} .
$$

Hence by Sobolev embedding we deduce that

$$
\begin{gathered}
\text { for } i \in K_{1} \quad \text { and } k>k_{0}, \\
\alpha \delta_{0}\left\|(\xi-k)^{+}\right\|_{L^{2}\left(W_{i}\right)} \leqslant\left\|\nabla(\xi-k)^{+}\right\|_{L^{1}\left(W_{i}\right)},
\end{gathered}
$$

where $\delta_{0}$ is an absolute constant.

Next we consider $K_{2}$. We deduce from step 1 that there is some $\delta_{1}$ in $\left(\delta_{0} / 2, \delta_{0}\right)$ such that

$$
V\left(k_{0}\right) \cap \Lambda\left(\delta_{1}\right)=\emptyset \text {. }
$$

It follows that, for $i \in K_{2}$,

$$
W_{i} \subset F\left(\delta_{1}\right) \subset F\left(\delta_{0}\right)
$$

Therefore for $i \in K_{2}$, we may consider the set

$$
\tilde{W}_{i}=W_{i} \cup T\left(W_{i}\right)
$$

and the function $\tilde{\xi}$ defined on $\tilde{W}_{i}$ by

$$
\begin{gathered}
\tilde{\xi}(x)=\xi(x) \quad \text { if } \quad x \in W_{i} \\
\tilde{\xi}(x)=\xi\left(T^{-1}(x)\right) \quad \text { if } \quad x \in \tilde{W}_{i} .
\end{gathered}
$$

We verify that $\tilde{\xi}$ is lipschitz and that $(\tilde{\xi}-k)^{+}=0$ on $\partial \tilde{W}_{i}$. Therefore

$$
\begin{gathered}
\text { for } i \in K_{2}, k>k_{0}, \\
\alpha \delta_{0}\left\|(\tilde{\xi}-k)^{+}\right\|_{L^{2}\left(\tilde{W}_{i}\right)} \leqslant\left\|\nabla(\tilde{\xi}-k)^{+}\right\|_{L^{1}\left(\tilde{W}_{i}\right)} .
\end{gathered}
$$

On the other hand, we have

$$
\left\|(\tilde{\xi}-k)^{+}\right\|_{L^{2}\left(\tilde{W}_{i}\right)} \geqslant\left\|(\xi-k)^{+}\right\|_{L^{2}\left(W_{i}\right)}
$$

and

$$
\left\|\nabla(\tilde{\xi}-k)^{+}\right\|_{L^{1}\left(\tilde{W}_{i}\right)} \leqslant C\left\|\nabla(\xi-k)^{+}\right\|_{L^{1}\left(W_{i}\right)} .
$$

Combining (V.19), (V.20), (V.21) we obtain

$$
\begin{gathered}
\text { for } i \in K_{2} \quad k>k_{0}, \\
C \alpha \delta_{0}\left\|(\xi-k)^{+}\right\|_{L^{2}\left(W_{i}\right)} \leqslant\left\|\nabla(\xi-k)^{+}\right\|_{L^{1}\left(W_{i}\right)} .
\end{gathered}
$$

Finally combining (V.16) and (V.22) we obtain (V.15). 
Step 3. - We complete the proof of proposition V.3 as in Stampacchia [15]. Applying (V.10) with the test function $\phi=(\xi-k)^{+}$we obtain

$$
\gamma \int_{\Omega}\left|\nabla(\xi-k)^{+}\right|^{2}+\xi(\xi-k)^{+} \leqslant \int_{\Omega}\left|f . \nabla(\xi-k)^{+}\right|
$$

which yields, by Hölder inequality, (note that $\xi \cdot(\xi-k)^{+} \geqslant 0$ )

$$
\gamma\left\|\nabla(\xi-k)^{+}\right\|_{L^{2}(\Omega)} \leqslant\|f\|_{L^{2}\left(\Omega_{k}\right)} \leqslant \mu(k)^{\frac{1}{2}-\frac{1}{q}}\|f\|_{L^{q}(\Omega)} .
$$

Hence

$$
\left\|\nabla(\xi-k)^{+}\right\|_{L^{1}(\Omega)} \leqslant \frac{1}{\gamma} \mu(k)^{1-\frac{1}{q}}\|f\|_{L^{q}(\Omega)}
$$

By the result of step 2, we obtain for $k>k_{0}$

$$
\left\|(\xi-k)^{+}\right\|_{L^{1}(\Omega)} \leqslant \frac{1}{\alpha \delta} \mu(k)^{\nu}\|f\|_{L^{q}(\Omega)}
$$

where $\nu=\frac{3}{2}-\frac{1}{q}$. We have

$$
\left\|(\xi-k)^{+}\right\|_{L^{1}(\Omega)}=-\int_{k}^{+\infty}(t-k) d \mu(t) \equiv H(k),
$$

and we are led to the differential inequality

$$
H^{\prime}(k)=-\mu(k) \leqslant-\left(\frac{H(k)}{\beta}\right)^{\frac{1}{\nu}}
$$

where

$$
\beta=\frac{1}{\gamma \delta}\|f\|_{L^{q}}
$$

integrating, we obtain

$$
H(k)=0 \quad \text { for } \quad k \geqslant \beta^{\frac{1}{\nu}} \frac{\nu}{\nu-1} H\left(k_{0}\right)^{1-\frac{1}{\nu}}+k_{0} .
$$

On the other hand, by (V.23), we have

$$
H\left(k_{0}\right)=\left\|\left(\xi-k_{0}\right)^{+}\right\|_{L^{1}(\Omega)} \leqslant \beta|\Omega|^{\nu}
$$

Hence

$$
H(k)=0 \quad \text { for } \quad k \geqslant \beta \frac{\nu}{\nu-1}|\Omega|^{\nu-1}+k_{0},
$$


Which shows that

$$
\left\|\xi^{+}\right\|_{L^{\infty}} \leqslant \frac{1}{\gamma \delta} \frac{\nu}{\nu-1}|\Omega|^{\frac{1}{2}-\frac{1}{q}}\|f\|_{L^{q}}+2 D_{1}\|f\|_{L^{2}} \leqslant C|\Omega|^{\frac{1}{2}-\frac{1}{q}}\|f\|_{L^{q}}
$$

This proves proposition V.3, since a similar estimate holds for $\xi^{-}$.

\subsection{Proof of theorem V.I}

We divide the proof in several steps.

Step 1. - Proof of (V.1). Let $\eta$ be a smooth function defined on $\Omega$ such that

$$
\begin{gathered}
\eta \equiv 1 \quad \text { on } \quad \hat{\Omega}=\Omega \backslash \bigcup_{i \in J} B\left(x_{i}, 2 \lambda \epsilon\right), \\
\eta \equiv 0 \quad \text { on } \bigcup_{i \in J} B\left(x_{i}, \lambda \epsilon\right) \cap \Omega, \\
|\nabla \eta| \leqslant \frac{C}{\epsilon}
\end{gathered}
$$

where $C$ is a constant depending on $\Omega$. Let $a$ be the function defined on $\Omega$ by

$$
\begin{cases}a=\frac{1}{\rho^{2}} & \text { on } \quad \tilde{\Omega}=\Omega \backslash \bigcup_{i \in J} B\left(x_{i}, \lambda \epsilon\right) \\ a=1 & \text { on } \quad B\left(x_{i}, \lambda \epsilon\right) \cap \Omega .\end{cases}
$$

Thus we have $1 \leqslant a \leqslant 4$. Let $q$ be such that $\frac{1}{p}+\frac{1}{q}=1$ and $f$ be any function in $L^{q}(\Omega)$ such that

$$
f \equiv 0 \quad \text { on } \quad \Omega \cap B\left(x_{i}, 2 \lambda \epsilon\right) .
$$

Consider the solution $\xi$ to (V.10). In (V.10) we use the test function $\phi=\eta h$. This yields

$$
\int_{\Omega} a \nabla \xi \nabla(\eta h)+\xi \eta h=\int_{\Omega} f \nabla(\eta h)=\int_{\Omega} f \nabla h
$$

where we have used (V.24) and (V.28). We have

$$
\int_{\Omega} a \nabla \xi \nabla(\eta h)=\int_{\Omega} a \eta \nabla \xi \nabla h+\int_{\Omega} a h \nabla \xi \nabla \eta
$$

and

$$
\begin{aligned}
\int_{\Omega} a \eta \nabla \xi \nabla h & =\int_{\Omega} \frac{1}{\rho^{2}} \eta \nabla \xi \nabla h \\
& =\int_{\Omega} \frac{1}{\rho^{2}} \nabla(\xi \eta) \nabla h-\int_{\Omega} \frac{1}{\rho^{2}} \xi \nabla \eta \nabla h
\end{aligned}
$$


Recall that

$$
|\nabla h| \leqslant \frac{C}{\epsilon}
$$

Hence

$$
\begin{aligned}
\left|\int_{\Omega} \frac{1}{\rho^{2}} \xi \nabla \eta \nabla h\right| & \leqslant C \sum_{i \in J}\left(\int_{B\left(x_{i}, 2 \lambda \epsilon\right)} \frac{1}{\epsilon^{2}}\right)\|\xi\|_{L^{\infty}(\Omega)} \\
& \leqslant C\|\xi\|_{L^{\infty}(\Omega)} .
\end{aligned}
$$

By proposition V.2, we may write

$$
\begin{aligned}
\int_{\Omega} \frac{1}{\rho^{2}} \nabla(\xi \eta) \nabla h+\xi \eta h & =\int_{\partial \Omega} \frac{1}{\rho^{2}} \frac{\partial h}{\partial \nu}(\xi \eta) \\
& =-\int_{\partial \Omega} \frac{1}{\rho^{2}} g(\xi \eta)
\end{aligned}
$$

Hence

$$
\left|\int_{\Omega} \frac{1}{\rho^{2}} \nabla(\xi \eta) \nabla h+\xi \eta h\right| \leqslant C\|\xi\|_{L^{\infty}(\Omega)} .
$$

Finally, it remains to estimate

$$
\int_{\Omega} a h \nabla \xi \nabla \eta=\sum_{i \in J} \int_{B\left(x_{i}, 2 \lambda \epsilon\right)} a h \nabla \xi \nabla \eta .
$$

Set $h_{i}=h\left(x_{i}\right)$. We have by (V.32) (proposition II.6),

$$
\left|h-h_{i}\right| \leqslant C \quad \text { on } B\left(x_{i}, 2 \lambda \epsilon\right) \text {. }
$$

Hence, using (V.12)

$$
\begin{aligned}
\left|\int_{B\left(\boldsymbol{x}_{i}, 2 \lambda \epsilon\right)} a\left(h-h_{i}\right) \nabla \xi \nabla \eta\right| & \leqslant C \int_{B\left(\boldsymbol{x}_{i}, 2 \lambda \epsilon\right)}|\nabla \xi||\nabla \eta| \\
& \leqslant C\left(\int_{B\left(\boldsymbol{x}_{i}, 2 \lambda \epsilon\right)}|\nabla \xi|^{2}\right)^{\frac{1}{2}} \\
& \leqslant C\|f\|_{L^{2}(\Omega)}
\end{aligned}
$$

On the other hand

$$
\int_{B\left(x_{i}, 2 \lambda \epsilon\right)} a h_{i} \nabla \xi \nabla \eta=\int_{\Omega} a \nabla \xi \nabla\left(\eta_{i}-1\right) h_{i},
$$


where $\eta_{i}$ is the map

$$
\begin{array}{lll}
\eta_{i} \equiv \eta & \text { on } & B\left(x_{i}, 2 \lambda \epsilon\right) \\
\eta_{i} \equiv 1 & \text { on } & \Omega \backslash B\left(x_{i}, 2 \lambda \epsilon\right) .
\end{array}
$$

By the equation (V.10), satisfied by $\xi$ we have

$$
\begin{aligned}
\int_{\Omega} a \nabla \xi \nabla\left(\left(\eta_{i}-1\right) h_{i}\right) & =-\int_{\Omega}\left(\eta_{i}-1\right) h_{i} \xi \\
& =-\int_{B\left(x_{i}, 2 \lambda \epsilon\right)}\left(\eta_{i}-1\right) h_{i} \xi
\end{aligned}
$$

Therefore

$$
\begin{aligned}
\left|\int_{\Omega} a \nabla \xi \nabla\left(\left(\eta_{i}-1\right) h_{i}\right)\right| & \leqslant\|\xi\|_{L^{\infty}}\left(\int_{B\left(x_{i}, 2 \lambda \epsilon\right)}|h|+C\right) \\
& \leqslant C\|\xi\|_{L^{\infty}},
\end{aligned}
$$

where we have used the proposition III.1 (the local estimates). Finally, combining (V.29), (V.30), (V.31), (V.33), (V.34), (V.35), and (V.36), we obtain

$$
\left|\int_{\Omega} f . \nabla h\right| \leqslant C\left(\|\xi\|_{L^{\infty}}+\|f\|_{L^{2}}\right),
$$

and hence by proposition V.3

$$
\left|\int_{\Omega} f . \nabla h\right| \leqslant C\|f\|_{L^{q}(\hat{\Omega})}
$$

Therefore, since $f$ was arbitrary, satisfying (V.28), we deduce by duality that

$$
\|\nabla h\|_{L^{p}(\hat{\Omega})} \leqslant C_{p} \quad \forall p<2 .
$$

(Recall that $\hat{\Omega}=\Omega \backslash \bigcup_{i \in J} B\left(x_{i}, 2 \lambda \epsilon\right)$ ). On the other hand, since $|\nabla h| \leqslant C / \epsilon$ we have

$$
\sum_{i \in J} \int_{B\left(x_{i}, 2 \lambda \epsilon\right)}|\nabla h|^{2} \leqslant C .
$$

Combining (V.37), and (V.38) we prove (V.1).

Step 2. - Proof of (V.2). We have

$$
\left|\int_{\Omega} h\right|=\left|\int_{\partial \Omega} A . \tau\right|=\left|2 \pi d-\int_{\partial \Omega} g\right|<C .
$$

Hence (V.2) follows from (V.1), (V.39) and Sobolev imbedding. 
VORTICES FOR A VARIATIONAL PROBLEM RELATED TO SUPERCONDUCTIVITY 285

Step 3. - Proof of (V.3).

We have

$$
-\star d h=\left(i u, d_{A} u\right)
$$

and

$$
\left(u, d_{A} u\right)=\frac{1}{2} d|u|^{2}=\frac{1}{2} d \rho^{2} .
$$

Therefore, we have, for $\rho \neq 0$,

$$
\left|\nabla_{A} u\right|^{2} \leqslant \frac{1}{\rho^{2}}\left(|\nabla h|^{2}+|\nabla \rho|^{2}\right) .
$$

It follows that

$$
\left|\nabla_{A} u\right| \leqslant C(|\nabla h|+|\nabla \rho|) \quad \text { in } \hat{\Omega}
$$

and, in order to prove (V.3), it suffices to establish that

$$
\int_{\Omega}|\nabla \rho|^{p} \leqslant C_{p} \quad \forall p<2,
$$

where $C_{p}$ depends only on $p$ and $\Omega$.

Proof of (V.41)

Set

$$
\bar{\rho}=\operatorname{Max}\left(\rho, 1-\frac{1}{|\log \epsilon|^{2}}\right),
$$

and consider the set

$$
K=\left\{x \in \Omega, \rho \geqslant 1-\frac{1}{1-1 / \log ^{2} \epsilon}\right\} .
$$

Clearly $\nabla \bar{\rho}=\nabla \rho$ on $K$ and $\nabla \bar{\rho}=0$ on $\Omega \backslash K$. Moreover since

$$
\frac{1}{\epsilon^{2}} \int_{K}(1-\rho)^{2} \leqslant C|\log \epsilon|
$$

we deduce

$$
\operatorname{meas}(\Omega \backslash K) \leqslant C \epsilon^{2}|\log \epsilon|^{5}
$$

Recall equation (II.6)

$$
-\Delta \rho^{2}=\frac{2}{\epsilon^{2}} \rho^{2}\left(1-\rho^{2}\right)-2\left|\nabla_{A} u\right|^{2} \quad \text { in } \Omega,
$$

Vol. $12, \mathbf{n}^{\circ} 3-1995$. 
and multiply it by $(1-\bar{\rho})$, this yields

$$
\begin{aligned}
\int_{K} 2 \rho|\nabla \rho|^{2} \leqslant \int_{\Omega} 2(1-\bar{\rho})\left|\nabla_{A} u\right|^{2} & \leqslant 2\|1-\bar{\rho}\|_{L^{\infty}} \int_{\Omega}\left|\nabla_{A} u\right|^{2} \\
& \leqslant 2\|1-\bar{\rho}\|_{L^{\infty}}|\log \epsilon|
\end{aligned}
$$

Since $0 \leqslant 1-\bar{\rho} \leqslant 1 /|\log \epsilon|^{2}$, we obtain

$$
\int_{K}|\nabla \rho|^{2} \leqslant \frac{C}{|\log \epsilon|^{2}} \longrightarrow 0 .
$$

On the other hand, by Hölder's inequality we have

$$
\begin{aligned}
\int_{\Omega \backslash K}|\nabla \rho|^{p} & \leqslant\left(\int_{\Omega \backslash K}|\nabla \rho|^{2}\right)^{p / 2} \operatorname{meas}(\Omega \backslash K)^{1-p / 2} \\
& \leqslant C \quad(|\log \epsilon|)^{p / 2}\left(\epsilon^{2}|\log \epsilon|^{5}\right) 1-p / 2 .
\end{aligned}
$$

Combining the two previous inequalities we get (V.41).

\section{CONVERGENCE OF $\left(u_{\epsilon_{n}}, A_{\epsilon_{n}}\right)$}

We may extract a subsequence $\epsilon_{n} \rightarrow 0(n \rightarrow+\infty)$ such that

$$
\begin{gathered}
\# J_{\epsilon_{n}}=\text { const }=N_{1}, \\
x^{\epsilon_{n}} \rightarrow l_{i} \in \bar{\Omega} \quad \forall i \in J .
\end{gathered}
$$

We cannot exclude, at this point, the possibility that some of the points $x^{\epsilon_{n}}{ }_{i}$ converge to the same limit. We denote by $a_{1}, \ldots, a_{N_{0}}$ the collection of distinct limits $l_{i}$ with $N_{0} \leqslant N_{1}$.

For sake of simplicity we will use sometimes the notation $h_{n}=h_{\epsilon_{n}}$, $A_{n}=A_{\epsilon_{n}}, u_{n}=u_{\epsilon_{n}}$. Recall that the gauge $\left(u_{n}, A_{n}\right)$ is choosen so that

$$
\left\{\begin{array}{lll}
d^{*} A_{n}=0 & \text { in } & \Omega \\
A_{n} . \nu=0 & \text { on } & \partial \Omega,{ }_{c} r
\end{array}\right.
$$

and that $A_{n}=\left(-\xi_{n_{x_{2}}}, \xi_{n_{x_{1}}}\right)$ where $\xi_{n}$ is a solution of

$$
\left\{\begin{array}{l}
\Delta \xi_{n}=h_{n} \\
\xi_{n}=0
\end{array} \text { on } \quad \text { in } \quad \Omega\right.
$$


We deduce from theorem V.1 and the $L^{p}$ bound on $\nabla h$, extracting a further subsequence if necessary, that there is some $h_{*} \in W^{1, p}(\Omega, \mathbb{R})$ such that

$$
h_{n} \rightarrow h_{*} \quad \text { weakly in } W^{1, p}(\Omega, \mathbb{R}) \quad \forall p<2 .
$$

It follows that there is some $A_{*}$ in $W^{2, p}\left(\Omega, \mathbb{R}^{2}\right)$ and $\xi_{*}$ in $W^{3, p}(\Omega, \mathbb{R})$ such that

$$
\begin{aligned}
A_{n}-A_{*} \quad \text { weakly in } W^{2, p}\left(\Omega, \mathbb{R}^{2}\right) & \forall p<2, \\
\xi_{n}-\xi_{*} \quad \text { weakly in } W^{3, p}(\Omega, \mathbb{R}) & \forall p<2 .
\end{aligned}
$$

Finally, we have by (V.3)

$$
\int_{\Omega}\left|\nabla_{A_{n}} u_{n}\right|^{p} \leqslant C_{p} \quad \forall p<2,
$$

which yields, in view of the following estimate, (which follows from (V.2)),

$$
\int_{\Omega}\left|A_{n}\right|^{q}+\int_{\Omega}\left|\nabla A_{n}\right|^{q} \leqslant C_{q} \quad \forall q<+\infty,
$$

that

$$
\int_{\Omega}\left|\nabla u_{n}\right|^{p} \leqslant C_{p} \quad \forall p<2 .
$$

Hence passing to a further subsequence if necessary, we may assume that there is some map $u_{*} \in W^{1, p}\left(\Omega, \mathbb{R}^{2}\right)$ such that

$$
u_{n}-u_{*} \quad \text { weakly in } W^{1, p}\left(\Omega, \mathbb{R}^{2}\right) \quad \forall p<2 .
$$

Clearly since

$$
\int_{\Omega}\left(1-\left|u_{n}\right|^{2}\right)^{2} \leqslant C \epsilon_{n}^{2}\left|\log \epsilon_{n}\right| \longrightarrow 0
$$

we obtain

$$
\left|u_{*}\right|=1 \quad \text { almost everywhere. }
$$

Proposition VI.1. - Let $K$ be a compact subset of $\bar{\Omega} \backslash \bigcup_{i=1}^{N_{0}}\left\{a_{i}\right\}$ we have

$$
\int_{K}\left|\nabla h_{n}\right|^{2}+\left|\nabla u_{n}\right|^{2}<C_{K}
$$

Vol. 12, $n^{\circ}$ 3-1995. 
where $C$ is some constant, and moreover

$$
\begin{aligned}
& h_{n} \rightarrow h_{*} \quad \text { strongly in } H^{1}(K), \\
& u_{n} \rightarrow u_{*} \quad \text { strongly in } H^{1}(K), \\
& \text { and } \quad \frac{1}{\epsilon_{n}^{2}} \int_{K}\left(1-\left|u_{n}\right|^{2}\right)^{2} \longrightarrow 0
\end{aligned}
$$

Proof. - The proof is similar to the proof of theorem X.2 of [2]. Let $\eta$ be a smooth function compactly supported in $\bar{\Omega} \backslash \bigcup_{i=1}^{N_{0}}\left\{a_{i}\right\}$ such that $\eta \equiv 1$ on $K$. Recall that on $\tilde{\Omega}_{\epsilon_{n}}, h_{n}$ verifies (see proposition V.2)

$$
-\operatorname{div}\left(\frac{1}{\rho_{n}^{2}} \nabla h_{n}\right)+h_{n}=0 \quad \text { in } \quad \tilde{\Omega}_{\epsilon_{n}}
$$

For $n$ large enough, the support of $\eta$ is contained in $\tilde{\Omega}_{\epsilon_{n}}$. Hence we may multiply (VI.17) by $\eta h_{n}$. Integrating we obtain

$$
\begin{aligned}
\int_{K}\left|\nabla h_{n}\right|^{2}+h_{n}^{2} & \leqslant C\left|\int_{K}\right| \nabla \eta|| \nabla h_{n}\left|h_{n}\right|+\int_{\partial \Omega}\left|\frac{\partial h_{n}}{\partial \nu}\right| \cdot\left|h_{n}\right| \\
& \leqslant C \int_{\Omega}\left|\nabla h_{n}\right|\left|h_{n}\right|+\int_{\partial \Omega}\left|h_{n}\right|
\end{aligned}
$$

Since $h_{n}$ is bounded in $W^{1, p}(\Omega)(\forall p<2)$ we have

$$
\int_{\Omega}\left|\nabla h_{n}\right|\left|h_{n}\right|<C
$$

Likewise, by the trace theorem,

$$
\int_{\partial \Omega}\left|h_{n}\right|^{q} \leqslant C_{q} \quad \forall q<+\infty
$$

Hence

$$
\int_{K}\left|\nabla h_{n}\right|^{2}+\left|h_{n}\right|^{2} \leqslant C
$$

Moreover, since $h_{n} \rightarrow h_{*}$ we deduce that

$$
\int_{K}\left|\nabla h_{*}\right|^{2}+h_{*}^{2} \leqslant C
$$


Next we multiply (VI.17) by $\eta\left(h_{n}-h_{*}\right)$. We obtain

$$
\begin{aligned}
\int_{\Omega} \frac{\eta}{\rho_{n}^{2}}\left|\nabla h_{n}\right|^{2}+h_{n} \eta\left(h_{n}-h_{*}\right)= & \int_{\Omega}\left(h_{*}-h_{n}\right) \frac{1}{\rho_{n}^{2}} \nabla h_{n} \nabla \eta \\
& +\int_{\Omega} \frac{1}{\rho_{n}^{2}} \eta \nabla h_{n} \nabla h_{*} .
\end{aligned}
$$

Since $h_{n} \rightarrow h_{*}$ weakly in $H^{1}(K)$ we deduce by Sobolev imbedding that

$$
\left\|h_{n}-h_{*}\right\|_{L^{q}(K)} \longrightarrow 0 \quad \forall q<+\infty .
$$

Hence

$$
\int_{\Omega} \frac{1}{\rho_{n}^{2}} \eta\left|\nabla h_{n}\right|^{2} \longrightarrow \int_{\Omega} \eta\left|\nabla h_{*}\right|^{2}
$$

Since $\rho \leqslant 1$, this implies, by lower semi-continuity that

$$
\nabla h_{n} \longrightarrow \nabla h_{*} \quad \text { strongly in } L^{2}(K)
$$

and establishes (V.14).

We turn now to (VI.15) and (VI.16). We consider the equation for $\rho_{n}$ (see (II.6)),

$$
-\Delta \rho_{n}+\rho_{n}^{-3}\left|\nabla h_{n}\right|^{2}=\frac{1}{\epsilon_{n}^{2}} \rho_{n}\left(1-\rho_{n}^{2}\right) \quad \text { in } \quad \Omega_{\epsilon_{n}} .
$$

Multiplying (VI.20) by $\eta\left(1-\rho_{n}\right)$ and integrating we obtain

$$
\begin{aligned}
\int_{\Omega} \eta & \left(\left|\nabla \rho_{n}\right|^{2}+\frac{1}{\epsilon_{n}^{2}} \rho_{n}\left(1-\rho_{n}\right)^{2}\left(1+\rho_{n}\right)\right) \\
\leqslant & \int_{\Omega_{\epsilon_{n}}} \eta \frac{\left(1-\rho_{n}\right)}{\rho_{n}^{3}}\left|\nabla h_{n}\right|^{2} \\
& +\int_{\Omega}\left|\nabla \eta \nabla \rho_{n}\left(1-\rho_{n}\right)\right| .
\end{aligned}
$$

Since $\rho_{n} \rightarrow 1$ and is bounded in $W^{1, p}$ we deduce from (V.14) and dominated convergence that the right hand side on (VI.21) goes to zero. Hence

$$
\int_{K}\left|\nabla \rho_{n}\right|^{2}+\frac{1}{\epsilon_{n}}\left(1-\rho_{n}^{2}\right)^{2} \longrightarrow 0
$$

this proves (VI.16). Moreover we have

$$
\begin{aligned}
\left|\nabla_{A_{n}} u_{n}\right|^{2} & =\frac{1}{\rho_{n}^{2}}\left(i u_{n}, \nabla_{A_{n}} u_{n}\right)^{2}+\left|\nabla \rho_{n}\right|^{2} \\
& =\frac{1}{\rho_{n}^{2}}\left|\nabla h_{n}\right|^{2}+\left|\nabla \rho_{n}\right|^{2} .
\end{aligned}
$$


Hence we deduce that

$$
\nabla_{A_{n}} u_{n} \longrightarrow \nabla_{A_{*}} u_{*} \quad \text { strongly in } L^{2}(K)
$$

and (VI.15) follows from the strong convergence of $A_{n}$ to $A_{*}$ in $L^{\infty}$ and (VI.23).

Proposition VI.2. - We have

$$
\frac{1}{\epsilon_{n}^{2}} \int_{\Omega}\left(1-\left|u_{n}\right|^{2}\right)^{2} \leqslant C
$$

where $C$ depends only on $g, d$ and $\Omega$.

Proof. - By proposition VII.1 below (which of course does not rely on (VI.24)), for all $i, a_{i}$ is not in $\partial \Omega$. Let $\mu>0$,be small, such that

$$
B\left(a_{i}, \mu\right) \cap B\left(a_{j}, \mu\right)=\emptyset \quad i \neq j \quad \text { and } \quad B\left(a_{i}, \mu\right) \cap \partial \Omega=\emptyset .
$$

In view of proposition VI.1 we may find, by Fubini's theorem, some $\mu_{n} \in(\mu, 2 \mu)$ such that, for $n$ large enough

$$
\int_{\partial B\left(a_{i}, \mu_{n}\right)} \frac{1}{\epsilon_{n}^{2}}\left(1-\left|u_{n}\right|^{2}\right)^{2}+\left|\nabla_{A_{n}} u_{n}\right|^{2}+\left|h_{n}\right|^{2} \leqslant C(\mu)
$$

where $C(\mu)$ depends only on $\mu, g, d$, and $\Omega$. Applying proposition II.4 to $\left(u_{n}, A_{n}\right)$ on the set $B\left(a_{i}, \mu\right) \cap \Omega$ we deduce

$$
\int_{B\left(a_{i}, \mu_{n}\right) \cap \Omega} \frac{1}{\epsilon_{n}^{2}}\left(1-\left|u_{n}\right|^{2}\right)^{2} \leqslant C+\int_{B\left(a_{i}, \mu_{n}\right) \cap \Omega}\left|h_{n}\right|^{2} \leqslant C .
$$

Hence (VI.24) follows from (VI.16) and (VI.26).

\section{PROPERTIES OF $\left(u_{*}, A_{*}\right)$}

Proposition VII.1. - We have

$$
\begin{gathered}
N_{0}=d \\
a_{i} \notin \partial \Omega \quad \forall i=1, \ldots, d \\
d_{i}=\operatorname{deg}\left(u_{*}, a_{i}\right)=1 \quad \forall i=1, \ldots, d .
\end{gathered}
$$


Proof. - The argument is readily the same as in [2]. We briefly sketch it. As in [2], consider a domain $\Omega^{\prime}$ such that $\Omega \subset \subset \Omega^{\prime}$. We are going to extend first $u_{n}$ to $\Omega^{\prime}$. Note that

$$
u_{n} \times \frac{\partial u_{n}}{\partial \tau}=g+A_{n} \cdot \tau
$$

and hence, by (VI.6)

$$
\int_{\partial \Omega}\left|\frac{\partial u_{n}}{\partial \tau}\right|^{2}<C
$$

It follows that there is some map which extends $u_{n}$ to $\Omega^{\prime}$ (which will still be denoted by $\left.u_{n}\right)$, such that $u_{n} \in H^{1}\left(\Omega^{\prime} \backslash \Omega, S^{1}\right)$ and

$$
\int_{\Omega^{\prime} \backslash \Omega}\left|\nabla u_{n}\right|^{2} \leqslant C
$$

Similarly, we extend $u_{*}$ to $\Omega^{\prime}$. We first establish (VII.3).

Let $\delta>0$, and consider the balls $B\left(a_{i}, \delta\right)$ and the domain $\Omega_{\delta}=$ $\Omega^{\prime} \backslash \bigcup_{i=1}^{N_{0}} B\left(a_{i}, \delta\right)$. by theorem I.8 of [2] we have

$$
\int_{\Omega_{\delta}}\left|\nabla u_{*}\right|^{2} \geqslant 2 \pi \sum_{i=1}^{N_{0}}\left|d_{i}\right|^{2} \log (1 / \delta)-C
$$

where $C$ depends only on the points $a_{i}$, and $g$. Hence, from the strong convergence (VI.15), we deduce

$$
\int_{\Omega_{\delta}}\left|\nabla u_{n}\right|^{2} \geqslant 2 \pi \sum_{i=1}^{N_{0}}\left|d_{i}\right|^{2} \log (1 / \delta)-C,
$$

for $n$ sufficiently large depending on $\delta$. On the other hand, it follows from a result of [5], that

$$
\int_{B\left(a_{i}, \delta\right)}\left|\nabla u_{n}\right|^{2} \geqslant 2 \pi\left|d_{i}\right| \log \left(\delta / \epsilon_{n}\right)-C .
$$

Combining (VII.4), (VII.5) and (VII.6), we are led to

$$
\int_{\Omega}\left|\nabla u_{n}\right|^{2} \geqslant 2 \pi \sum_{i=1}^{N_{0}}\left(\left|d_{i}\right|^{2} \log (1 / \delta)+\left|d_{i}\right| \log \left(\delta / \epsilon_{n}\right)\right)-C
$$

Vol. $12, \mathrm{n}^{\circ} 3-1995$. 
for any $\delta>0$, and $n$ sufficiently large (depending on $\rho$ ). We also have, by (VI.7)

$$
\int_{\Omega}\left|\nabla u_{n}\right|^{2}=\int_{\Omega}\left|\nabla u_{n}-i A_{n} u_{n}\right|^{2}+R_{n},
$$

where $R_{n}<C$. Combining (VII.7), (VII.8) and proposition II.5 we obtain (VII.3). Property (VII.2) can be derived similarly adapting the arguments of [2], theorem VI.2.

Proposition VII.2. - The magnetic field $h_{*}$ verifies the equation

$$
\left\{\begin{array}{l}
-\Delta h_{*}+h_{*}=2 \pi \sum_{i=1}^{d} \delta_{a_{i}} \quad \text { in } \Omega \\
\frac{\partial h_{*}}{\partial \nu}=-g \quad \text { on } \quad \partial \Omega .
\end{array}\right.
$$

Proof. - Let $K$ be some compact subset of $\Omega \backslash \bigcup_{i=1}^{d}\left\{a_{i}\right\}$. We easily verify that

$$
-\Delta h_{*}+h_{*}=0 \quad \text { on } K \text {. }
$$

Indeed we may pass to the limit in equation V.6, thanks to the $L^{p}$ bound for $\nabla h_{n}$. Let $\delta>0$, we have

$$
\begin{aligned}
\int_{\partial B\left(a_{i}, \delta\right)} & \frac{1}{\rho_{n}^{2}} \frac{\partial h_{n}}{\partial \nu} \\
= & -\int_{\partial B\left(a_{i}, \delta\right)} \frac{1}{\rho_{n}^{2}}\left(i u_{n}, \tau \cdot \nabla_{A_{n}} u_{n}\right) \\
& -\int_{\partial B\left(a_{i}, \delta\right)} \frac{1}{\rho_{n}^{2}}\left(i u_{n}, \tau \cdot \nabla u_{n}\right)+\int_{\partial B\left(a_{i}, \delta\right)} A_{n} \cdot \tau \\
= & -2 \pi d_{i}+\int_{B\left(a_{i}, \delta\right)} h=-2 \pi d_{i}-O(\delta)
\end{aligned}
$$

where $\nu$ is the exterior normal of $\partial B\left(a_{i}, \delta\right)$. By standard arguments, passing to the limit in (V.6) on the whole of $\Omega$, this implies (VII.9).

Proposition VII.3. - The map $u_{*}$ is a harmonic map on $\Omega \backslash \bigcup_{k=1}^{d}\left\{a_{k}\right\}$. i.e. verifies

$$
-\Delta u_{*}=u_{*}\left|\nabla u_{*}\right|^{2} \quad \text { on } \Omega \backslash \bigcup_{k=1 \ldots d}\left\{a_{k}\right\}
$$


VORTICES FOR A VARIATIONAL PROBLEM RELATED TO SUPERCONDUCTIVITY 293

Moreover we have

$$
u_{*}=\prod_{k=1 \ldots d} \frac{z-a_{k}}{\left|z-a_{k}\right|} e^{i \phi_{*}}
$$

where $\phi_{*}$ verifies (17) and (18) (in the introduction).

Proof. - Applying the operator $d^{*}$ to the equation (II.2), we obtain

$$
d^{*}\left[u_{n} \times d_{A_{n}} u_{n}\right]=0 \quad \text { in } \mathcal{D}^{\prime}(\Omega) .
$$

Passing to the limit in (VII.13), we are led to

$$
d^{*}\left[u_{*} \times d_{A_{*}} u_{*}\right]=0 \quad \text { in } \mathcal{D}^{\prime}(\Omega)
$$

Since $d^{*} A_{*}=0$, and $\left|u_{*}\right|=1$, we deduce that

$$
d^{*}\left[u_{*} \times d u_{*}\right]=0 \quad \text { in } \mathcal{D}^{\prime}(\Omega)
$$

Hence, we obtain (VII.11). Moreover, (VII.12) follows from (VII.11), the $L^{p}$ bounds of $u_{*}$ and the analysis of [2], section I.3. Finally combining the propositons of sections VI and VII we have proved theorem 3.

\section{RENORMALIZED ENERGIES}

The aim of this section is to prove Theorem 4, i.e. to determine the configuration $\left(a_{i}\right)$. For that purpose, we introduce an auxiliary problem and follow the strategy of [2], section VIII.

Consider $d$ points $b_{1}, \ldots, b_{d}$ in $\Omega$, and $\delta>0$. Clearly for $\delta$ sufficiently small (say $\delta<\delta_{0}$, depending on the configuration $\left(b_{i}\right)$ )

$$
\begin{gathered}
B\left(b_{i}, \delta\right) \subset \Omega \quad \forall i \in\{1, \ldots, d\} \\
B\left(b_{i}, \delta\right) \cap B\left(b_{j}, \delta\right)=\emptyset \quad \text { for } i \neq j .
\end{gathered}
$$

Set $\Omega_{\delta}=\Omega \backslash \bigcup_{i=1}^{d} B\left(a_{i}, \delta\right)$ and consider now the space $W_{\delta}$ defined by

$$
W_{\delta}=\left\{\begin{array}{c}
(u, A) \in H^{1}\left(\Omega_{\delta}, S^{1}\right) \times H^{1}\left(\Omega, \mathbb{R}^{2}\right), \text { such that } \\
\left(i u, \tau \cdot\left(\nabla_{A} u\right)\right)=g \text { on } \partial \Omega \\
\operatorname{deg}\left(u, \partial B\left(b_{i}, \delta\right)\right)=1
\end{array}\right\}
$$


and the functional $E_{\delta}$ defined on $W_{\delta}$ by

$$
E_{\delta}(u, A)=\frac{1}{2} \int_{\Omega_{\delta}}\left|\nabla_{A} u\right|^{2}+\frac{1}{2} \int_{\Omega}|d A|^{2} .
$$

Set

$$
\mu_{\delta}=\operatorname{Inf}_{(u, A) \in W_{\delta}} E_{\delta}(u, A)
$$

Proposition VIII.1. - We have: $\mu_{\delta}$ is achieved and for $\delta<\delta_{0}$

$$
\mu_{\delta} \leqslant \pi d \log \frac{1}{\delta}+C
$$

where $C$ depends only on the configuration $\left(b_{i}\right)$ and $\delta_{0}$.

Proof. - The proof is similar to the proof of proposition II.5, therefore we omit it.

Proposition VIII.2. - We have, for $\delta<\delta_{0}$, and for a minimizer $\left(v_{\delta}, B_{\delta}\right)$ of (VIII.2)

$$
\int_{\Omega}\left|d B_{\delta}\right|^{2} \leqslant C
$$

and

$$
\int_{\Omega_{\delta}}\left|\nabla_{B_{\delta}} v_{\delta}\right|^{2} \geqslant \pi d \log \frac{1}{\delta}-C,
$$

where $C$ depends only on $\left(b_{i}\right)$ on $\delta_{0}$.

Proof. - Let $\left(v_{\delta}, B_{\delta}\right)$ be a minimizer of (VIII.2) such that

$$
\left\{\begin{array}{lll}
d^{*} B=0 & \text { in } & \Omega \\
B \cdot \nu=0 & \text { on } & \partial \Omega .
\end{array}\right.
$$

(In this proof we omit the subscript $\delta$ for sake of simplicity). Hence we have

$$
B=\star d \xi
$$

where $\xi$ solves

$$
\left\{\begin{array}{l}
\Delta \xi=h=\star d B \quad \text { in } \Omega \\
\xi=0 \text { on } \partial \Omega .
\end{array}\right.
$$

We have

$$
\left.\int_{\Omega_{\delta}}|\nabla|_{B}\right|^{2}=\int_{\Omega_{b}}|\nabla v|^{2}+|\nabla \xi|^{2}+2[\xi, v]
$$


where we have used the notation $[\xi, v]=d \xi \wedge(i v, d v)$. We notice that

$$
\begin{aligned}
& \int_{\Omega_{\delta}} d \xi \wedge(i v, d v) \\
& =-\int_{\Omega_{\delta}} \xi d(i v, d v)+\sum_{i=1}^{d} \int_{\partial B\left(b_{i}, \delta\right)} \xi\left(i v, v_{\tau}\right) \\
& \quad=\sum_{i=1}^{d} \int_{\partial B\left(b_{i}, \delta\right)} \xi\left(i v, v_{\tau}\right)
\end{aligned}
$$

where $\tau$ is the unit tangential vector to $\partial B\left(b_{i}, \delta\right)$ such that $(\nu, \tau)$ is direct, $\nu$ is the exterior normal to $\partial B\left(b_{i}, \delta\right)$. Here we have used the fact that $d(i v, d v)=(i d v \wedge d v)=0$. Since $\operatorname{deg}\left(v, \partial B\left(b_{i}, \delta\right)\right)=1$, we may write

$$
v=e^{i(\theta+\psi)} \quad \text { on } \quad U_{i} \backslash B\left(b_{i}, \delta\right)
$$

where $U_{i}$ is some fixed neighborhood of $b_{i}$, and where $\psi$ is some (singled) valued function on $U_{i} \backslash B\left(b_{i}, \delta\right)$. We verify that, for $x \in U_{i} \backslash B\left(b_{i}, \delta\right)$

$$
|\nabla \psi|^{2} \leqslant 2\left(|\nabla v|^{2}+\frac{1}{\left|x-b_{i}\right|^{2}}\right)
$$

Integrating by parts, we obtain

$$
\int_{\partial B\left(b_{i}, \delta\right)} \xi\left(i v, v_{\tau}\right)=2 \pi \bar{\xi}_{i}+\int_{\partial B\left(b_{i}, \delta\right)} \xi_{\tau}\left(\psi-\bar{\psi}_{i}\right)
$$

where $\bar{\xi}_{i}=\frac{1}{\left|\partial B\left(b_{i}, \delta\right)\right|} \int_{\partial B\left(b_{i}, \delta\right)} \xi$ and $\bar{\psi}_{i}=\frac{1}{\left|\partial B\left(b_{i}, \delta\right)\right|} \int_{\partial B\left(b_{i}, \delta\right)} \psi$. By Sobolev imbedding and elliptic estimates, we have for any $\alpha<1$,

$$
|\xi|_{C^{0, \alpha}} \leqslant C_{\alpha}|\Delta \xi|_{L^{2}}
$$

On the other hand

$$
\int_{B\left(b_{i}, \delta\right)} \frac{\partial \xi}{\partial x_{j}}=\int_{\partial B\left(b_{i}, \delta\right)} \xi e_{j} \cdot \nu=\int_{\partial B\left(b_{i}, \delta\right)}\left(\xi-\xi\left(b_{i}\right)\right) e_{j} . \nu
$$

and by (VIII.10)

$$
\frac{1}{\left|B\left(b_{i}, \delta\right)\right|}\left|\int_{B\left(b_{i}, \delta\right)} \frac{\partial \xi}{\partial x_{j}}\right| \leqslant 2 \pi C_{\alpha} \delta^{\alpha-1}|\Delta \xi|_{L^{2}} .
$$


By the trace Theorem, we obtain

$$
\begin{aligned}
\int_{\partial B\left(b_{i}, \delta\right)}\left|\frac{\partial \xi}{\partial x_{j}}-\frac{1}{\left|B\left(b_{i}, \delta\right)\right|} \int_{B\left(b_{i}, \delta\right)} \frac{\partial \xi}{\partial x_{j}}\right|^{2} & \leqslant C \delta \int_{\Omega}\left|\nabla^{2} \xi\right|^{2} \\
& \leqslant C \delta \int_{\Omega}|\Delta \xi|^{2}
\end{aligned}
$$

Combining (VIII.11) and (VIII.12), we are led to

$$
\int_{\partial B\left(b_{i}, \delta\right)}|\nabla \xi|^{2} \leqslant C\left(\delta+\delta^{2 \alpha-1}\right) \int_{\Omega}|\Delta \xi|^{2} .
$$

We have also (by the trace Theorem and Sobolev imbedding)

$$
\begin{aligned}
\int_{\partial B\left(b_{i}, \delta\right)}\left|\psi-\bar{\psi}_{i}\right|^{2} & \leqslant C \delta \int_{U}|\nabla \psi|^{2} \\
& \leqslant C \delta\left(\int_{\Omega_{\delta}}|\nabla v|^{2}+2 \pi \log (1 / \delta)\right)
\end{aligned}
$$

combining (VIII.9), (VIII.13) and (VIII.14), we conclude

$$
\begin{aligned}
& \left|\int_{\partial B\left(b_{i}, \delta\right)} \xi_{\tau}\left(\psi-\bar{\psi}_{i}\right)\right| \\
& \quad \leqslant C \delta^{\alpha}|\Delta \xi|_{L^{2}(\Omega)}\left(|\nabla v|_{L^{2}\left(\Omega_{\delta}\right)}+(\log (1 / \delta))^{1 / 2}\right)
\end{aligned}
$$

Turning to the first term on the r.h.s. of (VIII.6), we have by a result of [2], theorem I.8.

$$
\int_{\Omega_{\delta}}|\nabla v|^{2} \geqslant 2 \pi d \log 1 / \delta-C
$$

Combining (VIII.6), (VIII.7), (VIII.9), (VIII.16), and proposition VIII.1, we obtain

$$
\left.\left|\int_{\Omega_{\delta}}\right| \Delta \xi\right|^{2}+|\nabla \xi|^{2}+4 \pi \sum_{i=1}^{d} \xi\left(b_{i}\right)+R(\delta) \mid \leqslant C
$$

where

$$
R(\delta)=2 \sum_{i=1}^{d}\left[\left(\bar{\xi}_{i}-\xi\left(b_{i}\right)\right)+\int_{\partial B\left(b_{i}, \delta\right)} \xi_{\tau}\left(\psi-\bar{\psi}_{i}\right)\right] .
$$


Combining (VIII.15) and (VIII.10) we verify that

$$
|R(\delta)| \leqslant C|| \Delta \xi \|_{L^{2}(\Omega)}\left(\delta^{\alpha}|\nabla v|_{L^{2}\left(\Omega_{\delta}\right)}+\delta^{\alpha / 2}\right)
$$

Since $\nabla v=\nabla_{B} v+i v B$ we obtain

$$
\begin{aligned}
\int_{\Omega_{\delta}}|\nabla v|^{2} & \leqslant 2\left[\int_{\Omega_{\delta}}\left|\nabla_{B} v\right|^{2}+|B|^{2}\right] \\
& \leqslant 2\left[\int_{\Omega_{\delta}}\left|\nabla_{B} v\right|^{2}+|\nabla \xi|^{2}\right] \leqslant C \log (1 / \delta)
\end{aligned}
$$

(by proposition VIII.1). Going back to (VIII.19) we deduce that

$$
R(\delta) \longrightarrow 0 \quad \text { as } \quad \delta \rightarrow 0 .
$$

Since, by standard estimates

$$
\left|\sum_{i=1}^{d} \xi\left(b_{i}\right)\right| \leqslant C|\Delta \xi|_{L^{2}(\Omega)}
$$

(VIII.21), (VIII.22) and (VIII.117) yield

$$
|\Delta \xi|_{L^{2}(\Omega)} \leqslant C
$$

(VIII.4) and (VIII.5) follow.

Proposition VIII.3. - Let $\xi_{*}$ be the solution of (20). We have $\xi_{\delta} \rightarrow \xi_{*}$ in $W^{2,2}(\Omega)$ as $\delta \rightarrow 0$.

Proof. - We have on $\Omega_{\delta}$, by minimality of $\left(v_{\delta}, B_{\delta}\right)$

$$
\begin{cases}-\Delta^{2} \xi_{\delta}+\Delta \xi_{\delta}=0 & \text { in } \quad \Omega \\ \frac{\partial}{\partial \nu} \Delta \xi_{\delta}=-g & \text { on } \quad \partial \Omega .\end{cases}
$$

On the other hand

$$
\int_{\partial B\left(a_{i}, \delta\right)} \frac{\partial}{\partial \nu} \Delta \xi_{\delta}=-\int_{\partial B\left(a_{i}, \delta\right)}\left(i v_{\delta}, \tau \cdot \nabla_{B} v_{\delta}\right)=-2 \pi+o(1) .
$$

From proposition VIII.2 we know that

$$
\left|\Delta \xi_{\delta}\right|_{L^{2}(\Omega)}+\left|\nabla \xi_{\delta}\right|_{L^{2}(\Omega)} \leqslant\left|\Delta \xi_{\delta}\right|_{L^{2}(\Omega)} \leqslant C
$$

Vol. $12, n^{\circ} 3-1995$. 
and hence (passing to a subsequence if necessary) we may assert that

$$
\xi_{\delta} \rightarrow \bar{\xi} \quad \text { in } W^{2,2}(\Omega) \text { weakly. }
$$

Passing to the limit in (VIII.23) and using (VIII.24), we see that $\bar{\xi}$ verifies (20) and hence $\bar{\xi}=\xi_{*}$ (and the full sequence converges by uniqueness of the limit). By minimality of $\left(v_{\delta}, B_{\delta}\right)$ we have

$$
E_{\delta}\left(v_{\delta}, B_{\delta}\right) \leqslant E_{\delta}\left(v_{\delta}, A_{*}\right)
$$

that is

$$
\int_{\Omega_{\delta}}\left|\nabla_{B_{\delta}} v_{\delta}\right|^{2}+\int_{\Omega}\left|d B_{\delta}\right|^{2} \leqslant \int_{\Omega_{\delta}}\left|\nabla_{A_{*}} v_{\delta}\right|^{2}+\int_{\Omega}\left|d A_{*}\right|^{2} .
$$

Arguing as for (VIII.6) we obtain

$$
\begin{aligned}
\int_{\Omega_{\delta}}\left|d B_{\delta}\right|^{2}+\left|\nabla \xi_{\delta}\right|^{2} & +2\left[\xi_{\delta}, v_{\delta}\right] \\
& \leqslant \int_{\Omega}\left|d A_{*}\right|^{2}+\left|\nabla \xi_{*}\right|^{2}+2\left[\xi_{*}, v_{*}\right] .
\end{aligned}
$$

Since $\xi_{\delta} \rightarrow \xi_{*}$ weakly in $W^{2,2}(\Omega)$, we obtain

$$
\int_{\Omega}\left|d B_{\delta}\right|^{2} \leqslant \int_{\Omega}\left|d A_{*}\right|^{2}+o(1)
$$

that is

$$
\int_{\Omega}\left|\Delta \xi_{\delta}\right|^{2} \leqslant \int_{\Omega}\left|d \xi_{*}\right|^{2}+o(1)
$$

That implies the strong convergence of $\Delta \xi_{\delta}$ to $\Delta \xi_{*}$ in $L^{2}$ and Proposition VIII.3 follows.

Proposition VIII.4. - Let

$$
\bar{\mu}_{\delta}=\operatorname{Min}\left\{\begin{array}{c}
\frac{1}{2} \int_{\Omega_{\delta}}|\nabla u|^{2}, \quad u \in H^{1}\left(\Omega_{\delta}, S^{1}\right), \operatorname{deg}\left(u, \partial B\left(b_{i}, \delta\right)\right)=1 \\
\frac{\partial u}{\partial \tau}=i\left(g+\frac{\partial \xi_{*}}{\partial \nu}\right) u \quad \text { on } \quad \partial \Omega
\end{array}\right\} .
$$

We have

$$
\left.\left|\frac{1}{2} \int_{\Omega_{\delta}}\right| \nabla v_{\delta}\right|^{2}-\bar{\mu}_{\delta} \mid \longrightarrow 0 \text { as } \delta \rightarrow 0
$$


Proof. - Since

$$
\frac{\partial v_{\delta}}{\partial \tau}=i\left(g+\frac{\partial \xi_{\delta}}{\partial \nu}\right) v_{\delta} \quad \text { on } \quad \partial \Omega
$$

we see easily (by proposition VIII.3 and standard elliptic estimates) that

$$
\frac{1}{2} \int_{\Omega_{\delta}}\left|\nabla v_{\delta}\right|^{2} \geqslant \bar{\mu}_{\delta}+o(1)
$$

Recall

$$
R(\delta) \longrightarrow 0 \quad \text { as } \quad \delta \rightarrow 0
$$

We deduce

$$
\begin{aligned}
E_{\delta}\left(v_{\delta}, B_{\delta}\right)= & \frac{1}{2} \int_{\Omega_{\delta}}\left|\nabla v_{\delta}\right|^{2}+\frac{1}{2} \int_{\Omega}\left(\left|\Delta \xi_{*}\right|^{2}+\left|\nabla \xi_{*}\right|^{2}\right) \\
& +2 \pi \sum_{i=1}^{d} \xi_{*}\left(b_{i}\right)+o(1) .
\end{aligned}
$$

Hence, in view of (VIII.28), we have uncoupled $v$ and $B$, and roughly speaking, in order to minimize $E_{\delta}(v, B)$, it suffices to minimize $\bar{\mu}_{\delta}$, and this yields

$$
\frac{1}{2} \int_{\Omega_{\delta}}\left|\nabla v_{\delta}\right|^{2} \leqslant \bar{\mu}_{\delta}+o(1)
$$

Proposition VIII.4 follows.

PROPOSITION VIII.5. - Let $1<p<2$ the map $v_{\delta}$ remains bounded in $W^{1, p}$ and $v_{\delta}$ converges to $v_{*}$ strongly in $W^{1, p}$, and in $C_{l o c}^{k}\left(\Omega \backslash \bigcup_{k=1}^{d}\left\{a_{k}\right\}\right)$, where $v_{*}$ is defined on $\Omega$ by

$$
v_{*}=\prod_{k=1 \ldots d} \frac{z-a_{k}}{\left|z-a_{k}\right|} e^{i \phi_{*}}
$$

and where $\phi_{*}$ is harmonic and verifies

$$
\frac{\partial \phi_{*}}{\partial \tau}=-\left(i \prod_{k=1 \ldots d} \frac{z-a_{k}}{\left|z-a_{k}\right|}, \frac{\partial}{\partial \tau}\left(\frac{z-a_{k}}{\left|z-a_{k}\right|}\right)\right)+\frac{\partial \xi_{*}}{\partial \nu}+g .
$$


Proof. - Since $v_{\delta}$ takes its values into $S^{1}$ we have

$$
d^{*}\left(i v_{\delta}, \star d v_{\delta}\right)=0 \text { in } \Omega_{\delta}
$$

Let $\Phi$ be the solution of

$$
\left\{\begin{array}{l}
\Delta \Phi=2 \pi \sum_{i=1}^{d} \delta_{b_{i}} \\
\frac{\partial \Phi}{\partial \nu}=g+\frac{\partial \xi_{\delta}}{\partial \nu} .
\end{array}\right.
$$

We have

$$
d^{*}\left(\left(i v_{\delta}, \star d v_{\delta}\right)+d \Phi\right)=0
$$

and

$\int_{\partial B\left(b_{i}, \delta\right)}\left(\left(i v_{\delta}, \star d v_{\delta}\right)+d \Phi\right) \cdot \nu=-\int_{\partial B\left(b_{i}, \delta\right)}\left(i v_{\delta}, \frac{\partial v_{\delta}}{\partial \tau}\right)+\int_{\partial B\left(b_{i}, \delta\right)} \frac{\partial \Phi}{\partial \nu}=0$.

Hence, there is some $H_{\delta} \in H^{1}\left(\Omega_{\delta}, I R\right)$, such that

$$
\left(i v_{\delta}, \star d v_{\delta}\right)=-d \Phi+d^{*} H_{\delta}
$$

It follows (see [2])

$$
\int_{\Omega_{\delta}}\left|\nabla v_{\delta}\right|^{2}=\int_{\Omega_{\delta}}|\nabla \Phi|^{2}+\int_{\Omega_{\delta}}\left|\nabla H_{\delta}\right|^{2}+o(1) .
$$

From [2], section I, we have

$$
\left.\left|\int_{\Omega_{\delta}}\right| \nabla \Phi\right|^{2}-\bar{\mu}_{\delta} \mid \longrightarrow 0 \quad \text { as } \quad \delta \rightarrow 0,
$$

and from proposition VIII.4 we deduce that

$$
\int_{\Omega_{\delta}}\left|\nabla H_{\delta}\right|^{2} \longrightarrow 0
$$

Hence,

$$
\left\|\left(i v_{\delta}, \star d v_{\delta}\right)+d \Phi\right\|_{L^{2}\left(\Omega_{\delta}\right)} \longrightarrow 0 \quad \text { as } \delta \rightarrow 0,
$$

and proposition VIII.5 follows from the analysis of [2], section I. 
VORTICES FOR A VARIATIONAL PROBLEM RELATED TO SUPERCONDUCTIVITY 301

PROPOSITION VIII.6. - We have for any configuration $\left(b_{i}\right)$

$$
\mu_{\delta}\left(b_{i}\right)=W\left(b_{i}\right)+\pi d \log (1 / \delta)+o(1) \quad \text { as } \quad \delta \rightarrow 0
$$

where $W$ is defined by (19), (20), (21) and (22).

Proof. - We deduce from propositions VIII.3, VIII.4, and VIII.5 that, by (VIII.28)

$$
\begin{aligned}
\mu_{\delta}\left(b_{i}\right)= & \frac{1}{2} \int_{\Omega_{\delta}}\left|\nabla v_{\delta}\right|^{2}+\int_{\Omega_{\delta}}\left|\Delta \xi_{*}\right|^{2}+\left|\nabla \xi_{*}\right|^{2} \\
& +2 \pi \sum_{i=1}^{d} \xi_{*}\left(b_{i}\right)+o(1) .
\end{aligned}
$$

Moreover equation (20), established in proposition VIII.3, easily implies

$$
\frac{1}{2} \int_{\Omega}\left|\Delta \xi_{*}\right|^{2}+\left|\nabla \xi_{*}\right|^{2}=-\pi \sum_{i=1}^{d} \xi_{*}\left(b_{i}\right)+\frac{1}{2} \int_{\partial \Omega} \Delta \xi_{*} \frac{\partial \xi_{*}}{\partial \nu} .
$$

(VIII.26) and the analysis of [2], section I (see Theorem I.7), imply

$$
\frac{1}{2} \int_{\Omega_{\delta}}\left|\nabla v_{\delta}\right|^{2}=\pi d \log (1 / \delta)+\omega(b, d, g)+O(\delta)
$$

where

$$
\begin{aligned}
\omega(b, d, g)= & -\pi \sum_{i \neq j} \log \left|b_{i}-b_{j}\right|-\pi \sum_{i=1}^{d} R\left(b_{i}\right) \\
& +\frac{1}{2} \int_{\partial \Omega} \frac{\partial \Phi}{\partial \nu} \Phi,
\end{aligned}
$$

$\Phi$ is a function verifying (VIII.29) and $R$ denotes

$$
R(x)=\Phi(x)-\sum_{i=1}^{d} \log \left|x-b_{i}\right| .
$$

Combining (VIII.32), (VIII.33) and (VIII.34) we obtain the desired result.

Proof of theorem 4. - Set

$I(\epsilon, \delta)=\operatorname{Min}\left\{\begin{array}{c}\frac{1}{2} \int_{B(\delta)}|\nabla v|^{2}+\frac{1}{4 \epsilon^{2}}\left(1-|v|^{2}\right)^{2}, \quad v \in H^{1}\left(B(\delta), \mathbb{R}^{2}\right) \\ v=e^{i \theta} \quad \text { on } \quad \partial B(\delta)\end{array}\right\}$. 
Let $\left(b_{i}\right)$ be a configuration of $d$ points. As in [2] we may construct a comparison function $v_{\epsilon, \delta}$ in $H^{1}(\Omega)$ such that

$$
v_{\epsilon, \delta}=v_{\delta} \quad \text { on } \quad \Omega_{\delta}
$$

and

$$
\frac{1}{2} \int_{B\left(b_{i}, \delta\right)}\left|\nabla v_{\epsilon, \delta}\right|^{2}+\frac{1}{4 \epsilon^{2}}\left(1-\left|v_{\epsilon, \delta}\right|^{2}\right)^{2} \leqslant I(\epsilon, \delta)+C(\delta)
$$

where

$$
|C(\delta)| \longrightarrow 0 \quad \text { as } \delta \rightarrow 0 .
$$

It follows that

$$
\begin{aligned}
G_{\epsilon}\left(v_{\epsilon}, A_{\epsilon}\right) & \leqslant G_{\epsilon}\left(v_{\epsilon, \delta}, A_{\epsilon}\right) \\
& \leqslant E_{\delta}\left(v_{\delta}, A_{*}\right)+d I(\epsilon, \delta)+C(\delta) \\
& \leqslant \mu_{\delta}\left(b_{i}\right)+d I(\epsilon, \delta)+C(\delta) \\
& \leqslant W\left(b_{i}\right)+\pi d \log (1 / \delta)+d I(\epsilon, \delta)+C(\delta),
\end{aligned}
$$

where we have used proposition VIII.6, and where $C(\delta) \rightarrow 0$ as $\delta \rightarrow 0$. On the other hand, arguing as in the proof of lemma VIII. 2 of [2], we have

$$
G_{\epsilon}\left(u_{\epsilon}, A_{\epsilon}\right) \geqslant d I(\epsilon, \delta)+W\left(a_{i}\right)+\pi d \log (1 / \delta)+C^{\prime}(\delta),
$$

where $C^{\prime}(\delta) \rightarrow 0$ as $\delta \rightarrow 0$. Combining (VIII.35) and (VIII.36) we obtain, letting $\delta \rightarrow 0$,

$$
W\left(a_{i}\right) \leqslant W\left(b_{i}\right) \quad \forall\left(b_{i}\right),
$$

and this proves theorem 4 .

\section{REFERENCES}

[1] F. Bethuel, H. Brezis and F. Hélein, Asymptotics for the minimization of a GinzburgLandau functional, Calculus of Variations, Vol. I, 1993, pp. 123-148.

[2] F. Bethuel, H. Brezis and F. Hélein, Ginzburg-Landau Vortices, Birkhäuser, 1993.

[3] F. Bethuel, H. BREzIS and F. HÉleIn, Limite singulière pour la minimisation de fonctionnelles du type Ginzburg-Landau, C. R. Acad. Sci. Paris, Vol. 314, 1992, pp. 891-895.

[4] F. Bethuel, H. Brezis and F. HÉlein, Tourbillons de Ginzburg-Landau et énergies renormalisées, to appear in C. R. Acad. Sci. Paris, 1993.

[5] H. Brezis, F. Merle and T. Riviekre, Quantization effects for $-\Delta u=u\left(1-|u|^{2}\right)$ in $\mathbb{R}^{2}$, to appear in Arch. for ratio. Mech., 1993. 
[6] H. Brezis, F. MerLe and T. Rivière, Quantifications pour les solutions de $-\Delta u=$ $u\left(1-|u|^{2}\right)$ dans $\boldsymbol{R}^{2}$, to appear in C. R. Acad. Sci. Paris, 1993.

[7] A. Boutet de Monvel-Berthier, V. Georgescu and R. Purice, Sur un problème aux limites de la théorie de Ginzburg-Landau, C. R. Acad. Sci. Paris, Vol. 307, 1988, pp. 55-58.

[8] A. Comtet and G. W. GibBons, Bogomol'nyi bounds for cosmic strings, Nucl. Phys. B, Vol. 299, 1988, pp. 719-733.

[9] Q. Du, M. Gunzburger and J. Peterson, Analysis and approximation of the GinzburgLandau model of superconductivity, SIAM Review, Vol. 34, 1992, pp. 45-81.

[10] P. GRIsvard, Elliptic Problems in non-smooth domains, Pitman, Marshfields, Mass, 1985.

[11] A. JAFFE and C. TAUBES, Vortices and Monopoles, Birkhäuser, 1980.

[12] D. Saint-James, G. Sarma and E. J. Thomas, Type II Superconductivity, Pergamon Press, 1969.

[13] J. SPRUCK and Y. YANG, Cosmic string solutions of the Einstein Matter gauge equations, to appear 1993.

[14] J. SpRUCK and Y. YANG, On multivortices in the electroweak theory II: existence of Bogomol'nyi solutions in $R^{2}$, Comm. Math. Phys., Vol. 144, 1992, pp. 215-234.

[15] G. STampacchia, Equations elliptiques du second ordre à coefficients discontinus, Presses Univ. de Montreal, 1966.

[16] Y. Yang, Boundary value problems of the Ginzburg-Landau equations, Proc. Roy. Soc. Edinburgh, Vol. 114 A, 1990, pp. 355-365.

(Manuscript received July 11, 1993;

Revised version received March 3, I994.) 